\title{
Expansion Trees with Cut
}

\author{
Federico Aschieri*, Stefan Hetzl’ and Daniel Weller \\ Institute of Discrete Mathematics and Geometry \\ Vienna University of Technology \\ Vienna, Austria \\ \{federico.aschieri, stefan.hetzl, daniel.weller\}@tuwien.ac.at
}

\begin{abstract}
Herbrand's theorem is one of the most fundamental insights in logic. From the syntactic point of view, it suggests a compact representation of proofs in classical first- and higher-order logic by recording the information of which instances have been chosen for which quantifiers.

This compact representation is known in the literature as Miller's expansion tree proof. It is inherently analytic and hence corresponds to a cut-free sequent calculus proof. Recently several extensions of such proof representations to proofs with cuts have been proposed. These extensions are based on graphical formalisms similar to proof nets and are limited to prenex formulas.

In this paper we present a new syntactic approach that directly extends Miller's expansion trees by cuts and covers also non-prenex formulas. We describe a cut-elimination procedure for our expansion trees with cut that is based on the natural reduction steps and show that it is weakly normalizing.
\end{abstract}

\section{Introduction}

Herbrand's theorem [14, 7], one of the most fundamental insights of logic, characterizes the validity of a formula in classical first-order logic by the existence of a propositional tautology composed of instances of that formula.

From the syntactic point of view this theorem induces a way of describing proofs: by recording which instances have been picked for which quantifiers we obtain a description of a proof up to its propositional part, a part we often want to abstract from. An example for a formalism that carries out this abstraction are Herbrand proofs [7]. This generalizes nicely to most classical systems with quantifiers, in particular to simple type theory, as in Miller's expansion tree proofs [20]. Such formalisms are compact and useful proof certificates in many situations; they are for example produced naturally by methods of automated deduction such as instantiation-based reasoning [18] and they play a central role in many proof transformations in the GAPT-system [11].

These formalisms consider only instances of the formula that has been proved and hence are analytic proof formalisms, corresponding to cut-free proofs in the sequent calculus. Considering an expansion tree to be a compact representation of a proof, it is thus natural to ask about the possibility of extending this kind of representation to non-analytic proofs, corresponding to proofs with cut in the sequent calculus.

In addition to enlarging the scope of instance-based proof representations, the addition of cuts to expansion trees promises to shed more light on the computational content of classical logic. This is a central topic of proof theory and has therefore attracted considerable attention, see $[22,2,10,9],[24,25]$, or [6], for different investigations in this direction.

\footnotetext{
${ }^{*}$ Funded by FWF Lise Meitner Grant M 1930-N35 and START project Y544-N23

${ }^{\dagger}$ Funded by the WWTF Vienna Research Group (VRG) 12-004
} 
Two proof formalisms manipulating only formula instances and incorporating a notion of cut have recently been proposed: proof forests [13] and Herbrand nets [19]. While some definitions in the setting of proof forests are motivated by the game semantics for classical arithmetic [8], Herbrand nets are based on methods for proof nets [12]. These two formalisms share a number of properties: both of them work in a graphical notation for proofs, both deal with prenex formulas only, for both weak but no strong normalization results are known.

In this paper we present a purely syntactic approach to the topic. We start from expansion tree proofs, add cuts and define cut-reduction rules, naturally extending the existing literature in this tradition. The result is a rewriting theory of expansion trees with cuts. The main staple of a good rewriting theory is that the syntax should look simple and the reduction rules should be as few and as elementary as possible. When a rewriting system falls short of any of these requirements, reasoning about its combinatorial properties may easily become unwieldy; when it satisfies them, it is always a good sign. Indeed, expansion trees are by design compact strings of symbols, expansion proofs just lists of those trees and the reduction rules that we shall present straightforward manipulation of those lists. This is a novel technical achievement. In fact, graph-based formalisms like proof forests and Herbrand nets allow rather simple mathematical definitions of tree forests and their transformations, but as soon as one tries to write them down syntactically, their rewriting complexity becomes evident. A simple rewriting theory may help to solve the intricate combinatorial problems that arise, like strong normalization.

With respect to proof forests, the main related work, we offer several technical novelties.

Miller's correctness criterion. Expansion trees are just simple collections of witnesses for quantifiers, so not every tree makes logical and semantical sense. Miller's correctness criterion [20] is the most direct known way to express that an expansion tree (list) is sound: it requires a certain acyclic ordering of the tree nodes, it maps the tree into a propositional formula and asks it to be a tautology. Syntactically, the definition of Miller's criterion follows in a straightforward way the tree's shape and the obtained propositional formula matches exactly the tree's number of leaves. Semantically, Miller's criterion states that a list of expansion trees represents a winning strategy in Coquand's backtracking games [8]. Though Heijltjes' correctness criterion was motivated as well by Coquand's game semantics, it represents a different way of extracting a propositional formula from a list of expansion trees: it is constructed from a case distinction on all cuts, rendering the formula's size exponential with the respect to the number of cuts. On our side, we managed to keep Miller's correctness criterion unchanged. As by-product, we also effortlessly obtain a treatment of non-prenex formulas. This avoids not only the distortion of the intuitive meaning of a formula by prenexification, but also the non-elementary increase in complexity that can be caused by prenexification [5]. It also seems possible to extend proof forests and Herbrand nets to non-prenex formulas, but this has not been done in [13].

Cut-Reductions. Eliminating cuts from expansion proofs resembles a Coquand game between expansion trees, when they are interpreted as strategies. Following this game semantics analogy, one would thus expect, during cut-elimination, to only encounter new trees whose branchings are isomorphic to sub-trees of the original expansion proof. This however does not happen in the theory of proof forests and Herbrand nets: the restructuring performed during cut-elimination is significant and trees eventually become much bigger than the original ones, due to an operation of copying and glueing them together. Though we are not pursuing the game semantics analogy here, we define cut-reduction steps that instead satisfy the mentioned condition. The gain is all about the rewriting theory of expansion proofs: cut-reduction only involves the operations of copying, decomposing, substituting terms and renaming variables 
applied to subtrees of the original ones.

Pruning and Bridges. In proof forests an unexpected technical issue arises. Cut-reductions create some unwanted "bridges" that cause non-termination of cut-elimination. Therefore, additional restructuring of the forest is needed, this time in terms of scissors, cutting those bridges. Here we show that bridges are not an issue at all and our cut-elimination terminates, regardless of bridges. Again, in this way we avoid an additional layer of rewriting complexity.

\subsection{Plan of the Paper}

In Section 2, we modify Miller's concept of expansion proof in order to also include special pairs of expansion trees, which represent logical cuts. In Section 3, we show that our expansion proofs are sound and complete with respect to first-order classical validity. In Section 4, we define a cut-elimination procedure which transform any expansion proof with cuts into a cut-free one.

\section{Expansion Trees}

In this entire paper we work with classical first-order logic. Formulas and terms are defined as usual. In order to simplify the exposition, we restrict our attention to formulas in negation normal form (NNF) and without vacuous quantifiers. Mutatis mutandis all notions and results of this paper generalize to arbitrary formulas. We write $\bar{A}$ for the de Morgan dual of a formula A. A literal is an atom $P\left(t_{1}, \ldots, t_{n}\right)$ or a negated atom $\bar{P}\left(t_{1}, \ldots, t_{n}\right)$. We start by defining Miller's concept of expansion tree [20].

Definition 1 (Expansion Trees). Expansion trees and a function $\mathrm{Sh}(\cdot)$ (for shallow) that maps an expansion tree to a propositional formula are defined inductively as follows:

1. A literal $L$ is an expansion tree with $\operatorname{Sh}(L)=L$.

2. If $E_{1}$ and $E_{2}$ are expansion trees and $\circ \in\{\wedge, \vee\}$, then $E_{1} \circ E_{2}$ is an expansion tree with $\operatorname{Sh}\left(E_{1} \circ E_{2}\right)=\operatorname{Sh}\left(E_{1}\right) \circ \operatorname{Sh}\left(E_{2}\right)$.

3. If $t_{1}, \ldots, t_{n}$ is a sequence of terms and $E_{1}, \ldots, E_{n}$ are expansion trees with $\operatorname{Sh}\left(E_{i}\right)=$ $A\left[t_{i} / x\right]$ for $i=1, \ldots, n$, then $E=\exists x A+{ }^{t_{1}} E_{1} \cdots+{ }^{t_{n}} E_{n}$ is an expansion tree with $\operatorname{Sh}(E)=\exists x A$.

4. If $E_{0}$ is an expansion tree with $\operatorname{Sh}\left(E_{0}\right)=A[\alpha / x]$, then $E=\forall x A+{ }^{\alpha} E_{0}$ is an expansion tree with $\operatorname{Sh}(E)=\forall x A$.

The $+^{t_{i}}$ of point 3. are called $\exists$-expansions and the $+^{\alpha}$ or point 4. are called $\forall$-expansions, and both $\forall$ - and $\exists$-expansions are called expansions. The variable $\alpha$ of $a \forall$-expansion $+^{\alpha}$ is called eigenvariable of this expansion. We say that $+^{t_{i}}$ dominates all the expansions in $E_{i}$. Similarly, $+{ }^{\alpha}$ dominates all the expansions in $E_{0}$. We also say that $E$ is an expansion tree of $\operatorname{Sh}(E)$. If $\mathcal{E}=E_{1}, \ldots, E_{n}$ is a sequence of expansion trees, we define $\operatorname{Sh}(\mathcal{E})=\operatorname{Sh}\left(E_{1}\right), \ldots, \operatorname{Sh}\left(E_{n}\right)$.

We recall now the definition of the propositional formula $\operatorname{Dp}(E)$, which is used to state Miller's correctness criterion for an expansion tree $E$. 
Definition 2. We define the function $\mathrm{Dp}(\cdot)$ (for deep) that maps an expansion tree to a propositional formula as follows:

$$
\begin{aligned}
\operatorname{Dp}(L) & =L \text { for a literal } L, \\
\operatorname{Dp}\left(E_{1} \circ E_{2}\right) & =\operatorname{Dp}\left(E_{1}\right) \circ \operatorname{Dp}\left(E_{2}\right) \text { for } \circ \in\{\wedge, \vee\}, \\
\operatorname{Dp}\left(\exists x A+{ }^{t_{1}} E_{1} \cdots+{ }^{t_{n}} E_{n}\right) & =\bigvee_{i=1}^{n} \operatorname{Dp}\left(E_{i}\right), \text { and } \\
\operatorname{Dp}\left(\forall x A+{ }^{\alpha} E_{0}\right) & =\operatorname{Dp}\left(E_{0}\right) .
\end{aligned}
$$

If $\mathcal{E}=E_{1}, \ldots, E_{n}$ is a sequence of expansion trees, we define $\operatorname{Dp}(\mathcal{E})=\operatorname{Dp}\left(E_{1}\right), \ldots, \operatorname{Dp}\left(E_{n}\right)$.

Cuts are simply defined as pairs of expansion trees, whose shallow formulas are one the involutive negation of the other.

Definition 3 (Cut). A cut is a set $C=\left\{E_{1}, E_{2}\right\}$ of two expansion trees s.t. $\operatorname{Sh}\left(E_{1}\right)=\overline{\operatorname{Sh}\left(E_{2}\right)}$. A formula is called positive if its top connective is $\vee$ or $\exists$ or a positive literal. An expansion tree $E$ is called positive if $\mathrm{Sh}(E)$ is positive. It will sometimes be useful to consider a cut as an ordered pair: to that aim we will write a cut as $C=\left(E_{1}, E_{2}\right)$ with parentheses instead of curly braces with the convention that $E_{1}$ is the positive expansion tree. For a cut $C=\left(E_{1}, E_{2}\right)$, we define $\operatorname{Sh}(C)=\operatorname{Sh}\left(E_{1}\right)$ which is also called cut-formula of $C$. We define $\operatorname{Dp}(C)=\operatorname{Dp}\left(E_{1}\right) \wedge \operatorname{Dp}\left(E_{2}\right)$. If $\mathcal{C}=C_{1}, \ldots, C_{n}$ is a sequence of cuts, we define $\operatorname{Dp}(\mathcal{C})=\operatorname{Dp}\left(C_{1}\right), \ldots, \operatorname{Dp}\left(C_{n}\right)$ and $\operatorname{Sh}(\mathcal{C})=$ $\operatorname{Sh}\left(C_{1}\right), \ldots, \operatorname{Sh}\left(C_{n}\right)$.

For each expansion tree we now define the set of finite formulas and number sequences, representing all formulas that one encounters and all branch choices one makes in any complete path from the tree's root to one of its leaves. This concept will soon be needed for defining correctness of expansion proofs.

Definition 4 (Formula Branch). We define a function $\operatorname{Br}(\cdot)$ (for branch) that maps an expansion tree with merges to a finite set $\left\{l_{1}, \ldots, l_{k}\right\}$, where each $l_{i}$ is some list made of formulas and the integers 1 or 2 .

$$
\begin{aligned}
\operatorname{Br}(L) & =L \text { for a literal } L, \\
\operatorname{Br}\left(E_{1} \circ E_{2}\right) & =\left\{\operatorname{Sh}\left(E_{1} \circ E_{2}\right), i, s \mid s \in \operatorname{Br}\left(E_{i}\right)\right\} \text { for } \circ \in\{\wedge, \vee\}, \\
\operatorname{Br}\left(\exists x A+{ }^{t_{1}} E_{1} \cdots+{ }^{t_{n}} E_{n}\right) & =\left\{\exists x A, s \mid s \in \operatorname{Br}\left(E_{i}\right) \text { with } i \in\{1, \ldots, n\}\right\}, \\
\operatorname{Br}\left(\forall x A+{ }^{\alpha} E_{0}\right) & =\left\{\forall x A, s \mid s \in \operatorname{Br}\left(E_{0}\right)\right\},
\end{aligned}
$$

For every cut $C=\left(E_{1}, E_{2}\right)$ we define $\operatorname{Br}(C)=\operatorname{Br}\left(E_{1}\right) \cup \operatorname{Br}\left(E_{2}\right)$.

A very simple property that we shall use without further mentioning is the following.

Lemma 1. Let $E$ be an expansion tree and $F$ a sub-tree of $E$. Then there is a formula sequence $s$ such that for every $r \in \operatorname{Br}(F)$, it holds that $s, r \in \operatorname{Br}(E)$.

Proof. By a straightforward induction on $E$.

Expansion proofs will be defined as sequences of expansion trees and cuts satisfying a number of properties. The correctness criterion of expansion tree proofs [20], as well as those of proof forests [13] and Herbrand nets [19], have two main components: 1. a tautology-condition 
on one or more quantifier-free formulas and 2. an acyclicity condition on one or more orderings. These conditions can be interpreted, logically, as ensuring that expansion proofs represents logical proofs, semantically, as defining correct winning strategies in Coquand games with backtracking $[13,1]$. While the tautology condition of [20] generalizes to the setting of cuts in a straightforward way, the acyclicity condition needs a bit more work: in the setting of cut-free expansion trees it is enough to require the acyclicity of an order on the $\exists$-expansions. In our setting that includes cuts we also have to speak about the order of cuts (w.r.t. each other and w.r.t. $\exists$-expansions). To simplify our treatment of this order we also include $\forall$-expansions. Together this leads to the following inference ordering constraints.

Definition 5 (Dependency Relation). Let $\mathcal{P}=\mathcal{C}, \mathcal{E}$, where $\mathcal{C}$ is a sequence of cuts and $\mathcal{E}$ a sequence of expansion trees. We will define the dependency relation $<_{\mathcal{P}}$, which is a binary relation on the set of expansions and cut occurrences in $\mathcal{P}$. First, we define the binary relation $<_{\mathcal{P}}^{0}$ (writing $<^{0}$ if $\mathcal{P}$ is clear from the context) as the least relation satisfying:

1. $v<^{0} w$ if $w$ is an $\exists$-expansion in $\mathcal{P}$ whose term contains the eigenvariable of the $\forall$ expansion $v$

2. $v<^{0} w$ if $v$ is an expansion in $\mathcal{P}$ that dominates the expansion $w$

3. $C<^{0} v$ if $v$ is an expansion of the cut $C$ in $\mathcal{C}$

4. $v<{ }^{0} C$ if $C$ is a cut and $\operatorname{Sh}(C)$ contains the eigenvariable of the $\forall$-expansion $v$

$<_{\mathcal{P}}$ is then defined to be the transitive closure of $<^{0}$. Again, we write $<_{\text {for }}<_{\mathcal{P}}$ if $\mathcal{P}$ is clear from the context.

As clauses 1-4 never relate two cuts, there is no $<_{\mathcal{P}}$-cycle containing cuts only, thus $<_{\mathcal{P}}$ is cyclic iff $w<_{\mathcal{P}} w$ for an expansion $w$ : we will make use of this property without further mention.

We now define the concept of expansion proof. In the following, lists of expansion trees and cuts will be identified modulo permutation of their elements.

Definition 6 (Expansion Proofs). Let $\mathcal{C}=C_{1}, \ldots, C_{n}$ be a sequence of cuts and let $\mathcal{E}=$ $E_{1}, \ldots, E_{m}$ be a sequence of expansion trees. Let $\mathcal{P}=\mathcal{C}, \mathcal{E}$. We define $\operatorname{Sh}(\mathcal{P})=\operatorname{Sh}(\mathcal{E})$, which corresponds to the end-sequent of a sequent calculus proof, and $\operatorname{Dp}(\mathcal{P})=\operatorname{Dp}(\mathcal{E}), \operatorname{Dp}(\mathcal{C})$, which is a sequent of quantifier-free formulas, and $\operatorname{Br}(\mathcal{P})=\operatorname{Br}\left(C_{1}\right) \cup \ldots \cup \operatorname{Br}\left(C_{n}\right) \cup \operatorname{Br}\left(E_{1}\right) \cup \ldots \cup$ $\operatorname{Br}\left(E_{m}\right)$. Then $\mathcal{P}$ is called expansion proof whenever:

1. (weak regularity) for every $S$ and $T$ in $\mathcal{P}$, if $s, \forall x A, A[\alpha / x], s^{\prime} \in \operatorname{Br}(T)$ and $r, \forall x B, B[\alpha / x], r^{\prime} \in$ $\operatorname{Br}(S)$, then $s=r, A=B$ and $S, T$ are both trees or both cuts.

2. (acyclicty) $<_{\mathcal{P}}$ is acyclic, that is, $x<_{\mathcal{P}} x$ holds for no $x$.

3. (validity) $\mathrm{Dp}(\mathcal{P})$ is a tautology, that is, a valid sequent.

4. (eigenvariable condition) For every $\forall$-expansion $+{ }^{\alpha}$ in $\mathcal{P}$, the variable $\alpha$ does not occur in $\operatorname{Sh}(\mathcal{E})$.

An important difference of expansion proofs with respect to other formalisms, such as proof forests [13] and Herbrand nets [19], is that the same $\forall$-expansion can occur multiple times. This phenomenon is very natural, as soon as one realizes that the weak regularity condition that we have imposed corresponds to an interpretation of eigenvariables as Skolem functions. Namely, weak regularity ensures: that the same witness is only used for the same formula with same parameters; that an expansion proof can always be transformed into one satisfying the usual regularity condition that every $\forall$-expansion occurs exactly one time [20]. This last property, that we shall not prove, guarantees that we are still working with the familiar objects. Our 
weak regularity condition offers, however, a great technical advantage. Namely, the definition of cut-reduction becomes much easier, as it avoids the heavy restructuring of the expansion trees which would be needed to prevent duplication of $\forall$-expansions.

Condition 2 and 3 embody Miller's correctness criterion. Condition 4 could be formulated as asking that $\operatorname{Sh}(\mathcal{P})$ does not contain free variables. But the real trouble is indeed that if $\mathcal{P}$ is such that $\operatorname{Sh}(\mathcal{P})$ contains free variables, then the eigenvariable of some $\forall$-expansion may be contained in $\operatorname{Sh}(\mathcal{P})$, so that $\mathcal{P}$ would not represent a proof of $\operatorname{Sh}(\mathcal{P})$. This issue will become transparent in Section 3, where we show that the notion of expansion proof represents indeed a sound and complete proof system with respect to classical first-order validity. Moreover, again because that the eigenvariable of some $\forall$-expansion could be contained in $\operatorname{Sh}(\mathcal{P})$, without condition 4 the notion of expansion proof would not be closed under the cut-reduction that we shall provide in Section 4.

Example 1. Consider the straightforward proof of $P(a) \rightarrow \exists z Q(z)$ from $\exists y \forall x(P(x) \rightarrow Q(f(y)))$ via a cut on $\forall x \exists y(P(x) \rightarrow Q(f(y)))$. In negation normal form these formulas are $\bar{P}(a) \vee \exists z Q(z)$, $\exists y \forall x(\bar{P}(x) \vee Q(f(y)))$, and $\forall x \exists y(\bar{P}(x) \vee Q(f(y)))$. The proof will be represented by the expansion proof $\mathcal{P}=\left\{E^{+}, E^{-}\right\}, E_{1}, E_{2}$ where

$$
\begin{aligned}
E^{+} & =\exists x \forall y(P(x) \wedge \bar{Q}(f(y)))+{ }^{a}\left(\forall y(P(a) \wedge \bar{Q}(f(y)))+{ }^{\gamma} P(a) \wedge \bar{Q}(f(\gamma))\right) \\
E^{-} & =\forall x \exists y(\bar{P}(x) \vee Q(f(y)))+{ }^{\beta}\left(\exists y(\bar{P}(\beta) \vee Q(f(y)))+{ }^{\alpha}(\bar{P}(\beta) \vee Q(f(\alpha)))\right) \\
E_{1} & =\forall y \exists x(P(x) \wedge \bar{Q}(f(y)))+{ }^{\alpha}\left(\exists x(P(x) \wedge \bar{Q}(f(\alpha)))+{ }^{\beta} P(\beta) \wedge \bar{Q}(f(\alpha))\right) \\
E_{2} & =\bar{P}(a) \vee\left(\exists z Q(z)+{ }^{f(\gamma)} Q(f(\gamma))\right)
\end{aligned}
$$

We have $\operatorname{Sh}(\mathcal{P})=\operatorname{Sh}\left(E_{1}, E_{2}\right)=\forall y \exists x(P(x) \wedge \bar{Q}(f(y))), \bar{P}(a) \vee \exists z Q(z)$ and

$$
\begin{aligned}
\operatorname{Dp}(\mathcal{P}) & =\operatorname{Dp}\left(E^{+}\right) \wedge \operatorname{Dp}\left(E^{-}\right), \operatorname{Dp}\left(E_{1}\right), \operatorname{Dp}\left(E_{2}\right) \\
& =(P(a) \wedge \bar{Q}(f(\gamma))) \wedge(\bar{P}(\beta) \vee Q(f(\alpha))), P(\beta) \wedge \bar{Q}(f(\alpha)), \bar{P}(a) \vee Q(f(\gamma))
\end{aligned}
$$

Note that $\operatorname{Dp}(\mathcal{P})$ is a tautology (of the form $A \wedge B, \bar{B}, \bar{A}$ ). Let us now consider the dependency relation induced by $\mathcal{P}$ : in $\mathcal{P}$ each term belongs to at most one $\exists$ - and at most one $\forall$-expansion. In such a situation we can uniformly write all expansions as $Q t$ for some term $t$ and $Q \in\{\exists, \forall\}$. The expansions of $\mathcal{P}$ are then written as $\exists a, \forall \gamma, \forall \beta, \exists \alpha, \forall \alpha, \exists \beta$, and $\exists f(\gamma)$. Furthermore, $\mathcal{P}$ contains a single cut $C$. Then $<^{0}$ is exactly:

1. $\forall \gamma<^{0} \exists f(\gamma), \forall \beta<^{0} \exists \beta, \forall \alpha<^{0} \exists \alpha$,

2. $\exists a<^{0} \forall \gamma, \forall \beta<<^{0} \exists \alpha, \forall \alpha<^{0} \exists \beta$,

3. $C<^{0} \exists a, C<^{0} \forall \gamma, C<^{0} \forall \beta, C<^{0} \exists \alpha$,

4. there is no $v<{ }^{0} C$ as the cut formula of $C$ is variable-free.

As the reader is invited to verify, < is acyclic.

\section{Expansion Proofs and Sequent Calculus}

In this section we will clarify the relationship between our expansion proofs and the sequent calculus. The concrete version of sequent calculus is of no significance to the results presented here, they hold mutatis mutandis for every version that is common in the literature. For technical convenience, we treat sequents as multisets of formulas in Section $\S 3.1$ and as sets of formulas in Section $§ 3.2$. 
Definition 7. The calculus $\mathbf{L K}$ is defined as follows: initial sequents are of the form $\Gamma, A, \bar{A}$ for an atom $A$. The inference rules are

$$
\frac{\Gamma, A[\alpha / x]}{\Gamma, \forall x A} \forall \frac{\Gamma, \exists x A, A[t / x]}{\Gamma, \exists x A} \exists \frac{\Gamma, A \quad \Gamma, B}{\Gamma, A \wedge B} \wedge \frac{\Gamma, A, B}{\Gamma, A \vee B} \vee \frac{\Gamma, A \quad \bar{A}, \Gamma}{\Gamma} \text { cut }
$$

with the usual side conditions: $\alpha$ must not appear in $\Gamma, \forall x A$ and $t$ must not contain a variable which is bound in A.

An LK-proof is called regular if each two $\forall$-inferences have different eigenvariables and different from the free variables in the conclusion of the proof. From now on we assume w.l.o.g. that all LK-proofs are regular.

\subsection{From Sequent Calculus to Expansion Proofs}

In this section we describe how to read off expansion trees from LK-proofs (with sequents as multisets), thus obtaining a completeness theorem for expansion proofs. For representing a formula $A$ that is introduced by (implicit) weakening we use the natural coercion of $A$ into an expansion tree, denoted by $A^{\mathrm{E}}:(\exists x A)^{E}=\exists x A+{ }^{x} A^{E},(\forall x A)^{E}=\forall x A+{ }^{\alpha} A^{E}$ ( $\alpha$ fresh), $\left(E_{1} \circ E_{2}\right)^{E}=E_{1}^{E} \circ E_{2}^{E}, L^{E}=L$ for $L$ atomic. For a sequent $\Gamma=A_{1}, \ldots, A_{n}$ we define $\Gamma^{\mathrm{E}}=A_{1}^{\mathrm{E}}, \ldots, A_{n}^{\mathrm{E}}$.

Definition 8. For an $\mathbf{L K}$-proof $\pi$ define the expansion proof $\operatorname{Exp}(\pi)$ by induction on $\pi$ :

1. If $\pi$ is an initial sequent $\Gamma, A, \bar{A}$, thus with $A$ atomic, then $\operatorname{Exp}(\pi)=\Gamma^{\mathrm{E}}, A, \bar{A}$

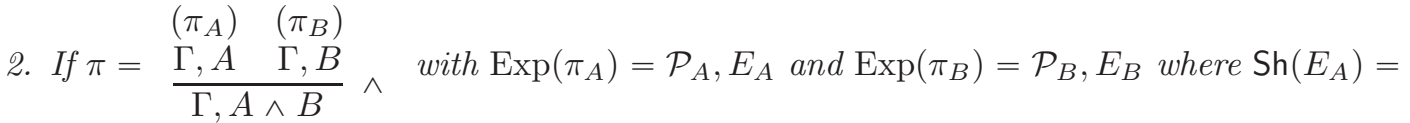
$A$ and $\operatorname{Sh}\left(E_{B}\right)=B$, then $\operatorname{Exp}(\pi)=\left(\mathcal{P}_{A}, \mathcal{P}_{B}, E_{A} \wedge E_{B}\right)$.

$\left(\pi^{\prime}\right)$

3. If $\pi=\frac{\Gamma, A, B}{\Gamma, A \vee B} \vee$ with $\operatorname{Exp}\left(\pi^{\prime}\right)=\mathcal{P}, E_{A}, E_{B}$ where $\operatorname{Sh}\left(E_{A}\right)=A$ and $\operatorname{Sh}\left(E_{B}\right)=B$, then $\operatorname{Exp}(\pi)=\left(\mathcal{P}, E_{A} \vee E_{B}\right)$.

$\left(\pi_{A}\right)$

4. If $\pi=\frac{\Gamma, A[\alpha / x]}{\Gamma, \forall x A} \forall$ with $\operatorname{Exp}\left(\pi_{A}\right)=\mathcal{P}, E$ where $\operatorname{Sh}(E)=A[\alpha / x]$, then $\operatorname{Exp}(\pi)=$ $\left(\mathcal{P}, \forall x A+{ }^{\alpha} E\right)$.

$\left(\pi_{A}\right)$

5. If $\pi=\frac{\Gamma, \exists x A, A[t / x]}{\Gamma, \exists x A} \exists$ with $\operatorname{Exp}\left(\pi_{A}\right)=\mathcal{P}, E, E_{t}$ where $\operatorname{Sh}(E)=\exists x A$ and $\operatorname{Sh}\left(E_{t}\right)=$ $A[t / x]$, then $\operatorname{Exp}(\pi)=\left(\mathcal{P}, E, \exists x A+{ }^{t} E_{t}\right)$.

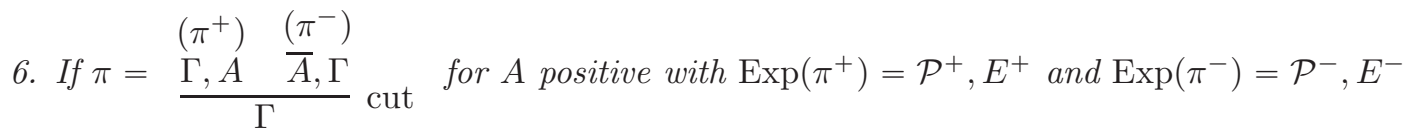
where $\operatorname{Sh}\left(E^{+}\right)=A$ and $\operatorname{Sh}\left(E^{-}\right)=\bar{A}$, then $\operatorname{Exp}(\pi)=\left(\left(E^{+}, E^{-}\right), \mathcal{P}^{+}, \mathcal{P}^{-}\right)$.

Theorem 2 (Completeness). If $\pi$ is an $\mathbf{L K}$-proof of a sequent $\Gamma$, then $\operatorname{Exp}(\pi)$ is an expansion proof such that $\operatorname{Sh}(\pi)=\Gamma$. If $\pi$ is cut-free, then so is $\operatorname{Exp}(\pi)$. 
Proof. That $\operatorname{Exp}(\pi)$ satisfies weak regularity and the eigenvariable condition follows directly from the definitions as we are dealing with regular LK-proofs only, thus we are constructing regular expansion proofs as well. By a straightforward induction on $\pi$ one shows that $\operatorname{Dp}(\operatorname{Exp}(\pi))$ is a tautology. Acyclicity is also shown inductively by observing that if $\alpha$ is a free variable in the end-sequent of $\pi$, then $\alpha$ is not an eigenvariable in $\operatorname{Exp}(\pi)$. This implies that if $w$ is the new expansion introduced in the construction of $\operatorname{Exp}(\pi)$, and $v$ is an old expansion in $\operatorname{Exp}(\pi)$, then $w \ngtr v$, which in turn yields acyclicity.

\subsection{From Expansion Proofs to Sequent Calculus}

In this section we show how to construct an LK-proof (with sequents as sets) from a given expansion proof. To this aim we introduce a calculus LKE, generalizing the treatment in [20], that works on sequences of expansion trees and cuts instead of sequents of formulas.

Definition 9. The axioms of $\mathbf{L K E}$ are of the form $\mathcal{P}, A, \bar{A}$ for an atom $A$. The inference rules are

$$
\begin{gathered}
\frac{\mathcal{P}, E_{1}, \ldots, E_{n}}{\mathcal{P}, \forall x A+{ }^{\alpha} E_{1}, \ldots, \forall x A+{ }^{\alpha} E_{n}} \\
\frac{\mathcal{P}, \exists x A+{ }^{t_{1}} E_{1} \cdots+{ }^{t_{n}} E_{n}+{ }^{t_{i}} E_{i}}{\mathcal{P}, \exists x A+{ }^{t_{1}} E_{1} \cdots+{ }^{t_{i}} E_{i} \cdots+{ }^{t_{n}} E_{n}} \exists \\
\frac{\mathcal{P}, \exists x A+{ }^{t_{1}} E_{1} \cdots+{ }^{t_{i}} E_{i}, \ldots, \exists x A+{ }^{s_{1}} F_{1} \cdots+{ }^{s_{j}} F_{j}, E_{i+1} \ldots, E_{n}, \ldots, F_{j+1}, \ldots, F_{m}}{\mathcal{P}, \exists x A+{ }^{t_{1}} E_{1} \cdots+{ }^{t_{n}} E_{n}, \ldots, \exists x A+{ }^{s_{1}} F_{1} \cdots+{ }^{s_{m}} F_{m}} \exists \\
\frac{\mathcal{P}, E_{1} \mathcal{P}, E_{2}}{\mathcal{P}, E_{1} \wedge E_{2}} \wedge \frac{\mathcal{P}, E_{1}, E_{2}}{\mathcal{P}, E_{1} \vee E_{2}} \vee \frac{\mathcal{P}, E_{1}, \ldots, E_{n}}{\mathcal{P},\left\{E_{1}, F_{1}\right\}, \ldots,\left\{E_{1}, \ldots, F_{n}, F_{n}\right\}} \text { cut }
\end{gathered}
$$

with the following side conditions: $\operatorname{Sh}\left(E_{1}\right)=\ldots=\operatorname{Sh}\left(E_{n}\right)$ for the cut rule; $t_{i+1}=\ldots=t_{n}=$ $\ldots=s_{j+1}=\ldots=s_{m}$ for the second $\exists$ rule; the eigenvariable condition for the $\forall$ rule: $\alpha$ must not occur in $\operatorname{Sh}(\mathcal{P}), \forall x A$.

The reader is invited to note that $\operatorname{Sh}(\mathcal{P}), \forall x A$ does not include the cut formulas of $\mathcal{P}$, though they may - and indeed often have to - contain the eigenvariable $\alpha$. An important feature of the above calculus, which is easily verified, is that if $\pi$ is an LKE-proof, then $\operatorname{Sh}(\pi)$ - defined as the result of replacing in $\pi$ each sequence $\mathcal{P}$ of expansion trees and cuts with $\operatorname{Sh}(\mathcal{P})-$ is a LK-proof. In the following proof we describe how to transform expansion proofs to LK-proofs.

Theorem 3 (Soundness). If $\mathcal{P}$ is an expansion proof of a sequent $\Gamma$, then there is an $\mathbf{L K}$-proof of $\Gamma$. If $\mathcal{P}$ is cut-free, then so is the $\mathbf{L K}$-proof.

Proof. It is enough to construct an LKE-proof $\pi$ of $\mathcal{P}$, as then $\operatorname{Sh}(\pi)$ is a proof of $\operatorname{Sh}(\mathcal{P})=\Gamma$. The construction will be carried out by induction on the number of nodes in $\mathcal{P}$. The inductive statement we are going to prove is: if $\mathcal{P}$ is an expansion proof, then there is a LKE-proof $\pi$ of $\mathcal{P}$.

If $\mathcal{P}$ contains only literals, the thesis is obvious.

If $\mathcal{P}=\mathcal{P}^{\prime}, E_{1} \vee E_{2}$ for some $\mathcal{P}^{\prime}, E_{1}$ and $E_{2}$, then $\mathcal{P}^{\prime}, E_{1}, E_{2}$ is a strictly smaller expansion proof. By the induction hypothesis we obtain an LKE-proofs $\pi^{\prime}$ of $\mathcal{P}^{\prime}, E_{1}, E_{2}$ from which a proof of $\mathcal{P}$ is obtained by an $\vee$-inference. For $\mathcal{P}=\mathcal{P}^{\prime}, E_{1} \wedge E_{2}$, proceed analogously.

So assume there are no top-level conjunctions or disjunctions. We observe that for any non-top-level quantifier expansion there is some top-level quantifier expansion that dominates it, and is smaller than it, according to $<_{\mathcal{P}}$. Thus, the $<_{\mathcal{P}}$-minimal quantifier expansions are all 
top-level. By the acyclicity of $<_{\mathcal{P}}$ there must be a $<_{\mathcal{P}}$-minimal quantifier expansion or cut. In the case the $<_{\mathcal{P}}$-minimal expression is a quantifier expansion, then it is a top-level one.

For the case of cut, we proceed as follows: let $C=\left\{E_{1}, \forall x A+{ }^{\alpha} F_{1}\right\}$ be a cut minimal with respect to $<_{\mathcal{P}}$ (if $\mathrm{Sh}(C)$ does not begin with a quantifier the argument is easier). Then $\forall x A, A[\alpha / x] \in \operatorname{Br}(C), s$, which, by weak regularity of $\mathcal{P}$, forces every element of $\mathcal{P}$ containing $+^{\alpha}$ to be a cut of the shape $\left\{E_{i}, \forall x A+{ }^{\alpha} F_{i}\right\}$. Then, we can write

$$
\mathcal{P}=\left\{E_{1}, \forall x A+{ }^{\alpha} F_{1}\right\}, \ldots,\left\{E_{n}, \forall x A+{ }^{\alpha} F_{n}\right\}, \mathcal{P}^{\prime}
$$

where $+{ }^{\alpha}$ does not occur in $\mathcal{P}^{\prime}$. Now,

$$
\operatorname{Dp}(\mathcal{P})=\left(\operatorname{Dp}\left(E_{1}\right) \wedge \mathrm{Dp}\left(F_{1}\right)\right) \vee \ldots \vee\left(\mathrm{Dp}\left(E_{n}\right) \wedge \mathrm{Dp}\left(F_{n}\right)\right) \vee \mathrm{Dp}\left(P^{\prime}\right)
$$

Therefore,

$$
\begin{gathered}
\operatorname{Dp}\left(E_{1}, \ldots, E_{n}, \mathcal{P}^{\prime}\right)=\operatorname{Dp}\left(E_{1}\right) \vee \ldots \vee \operatorname{Dp}\left(E_{n}\right) \vee \operatorname{Dp}\left(\mathcal{P}^{\prime}\right) \\
\operatorname{Dp}\left(\forall x A+{ }^{\alpha} F_{1}, \ldots, \forall x A+{ }^{\alpha} F_{n}, \mathcal{P}^{\prime}\right)=\operatorname{Dp}\left(F_{1}\right) \vee \ldots \vee \operatorname{Dp}\left(F_{m}\right) \vee \operatorname{Dp}\left(\mathcal{P}^{\prime}\right)
\end{gathered}
$$

are tautologies. To prove weak regularity of $E_{1}, \ldots, E_{n}, \mathcal{P}^{\prime}$ and $\forall x A+{ }^{\alpha} F_{1}, \ldots, \forall x A+{ }^{\alpha} F_{n}, \mathcal{P}^{\prime}$, we observe that $\operatorname{Br}\left(E_{1}, \ldots, E_{n}, \mathcal{P}^{\prime}\right)$ and $\operatorname{Br}\left(\forall x A+{ }^{\alpha} F_{1}, \ldots, \forall x A+{ }^{\alpha} F_{n}, \mathcal{P}^{\prime}\right)$ are contained in $\operatorname{Br}(\mathcal{P})$; the only issue is when a branch belongs to $\operatorname{Br}\left(\forall x A+{ }^{\alpha} F_{i}\right)$. In this case, in order to show weak regularity, we have to show that for every branch $b=s, \forall x A, A[\alpha / x], s^{\prime}$, we have that $s$ is empty and the branch $b$ is the branch of a tree. By weak regularity of $\mathcal{P}$, the same branch $b$ was in $\mathcal{P}$ a branch of a cut. Thus, it is enough to observe that $b$ belongs to some cut $\left\{E_{j}, \forall x A+{ }^{\alpha} F_{j}\right\}$, for some $j=i, \ldots, n$, otherwise $b$ would belong to some cut in $\mathcal{P}^{\prime}$, impossible by construction of $\mathcal{P}^{\prime}$. Furthermore, the orderings of the expansions and cuts of $E_{1}, \ldots, E_{n}, \mathcal{P}^{\prime}$ and $\forall x A+{ }^{\alpha} F_{1}, \ldots, \forall x A+{ }^{\alpha} F_{n}, \mathcal{P}^{\prime}$ are suborderings of $<_{\mathcal{P}}$, hence also acyclic. Last, $\operatorname{Sh}\left(E_{1}, \ldots, E_{n}, \mathcal{P}^{\prime}\right)$ and $\operatorname{Sh}\left(\forall x A+{ }^{\alpha} F_{1}, \ldots, \forall x A+{ }^{\alpha} F_{n}, \mathcal{P}^{\prime}\right)$ contain the same free variables of $\operatorname{Sh}(\mathcal{P})$ plus those of $\forall x A$; now, no $\forall$-expansion $+^{\beta}$ of $\mathcal{P}$ can have $\beta$ occur in $\forall x A$, otherwise $+{ }^{\beta}<_{\mathcal{P}} C$, contradicting the minimality assumption on $C$, so we have that the eigenvariable condition holds. Then, by the induction hypothesis we obtain LKE-proofs $\pi_{1}, \pi_{2}$ of $E_{1}, \ldots, E_{n}, \mathcal{P}^{\prime}$ and $\forall x A+{ }^{\alpha} F_{1}, \ldots, \forall x A+{ }^{\alpha} F_{n}, \mathcal{P}^{\prime}$ respectively, from which a proof of $\mathcal{P}$ is obtained by a cut.

For the case of the minimal node being an $\exists$-expansion, let $\exists x A \cdots+{ }^{t} E \cdots$ be an expansion tree of $\mathcal{P}$ such that $+{ }^{t}$ is minimal with respect to $<_{\mathcal{P}}$. As we said, $+^{t}$ occurs at top-level. We move all top-level $+^{t}$ at the end of the lists of $\exists$-espansions relative to the corresponding top level formula. In this way, we can rewrite $\mathcal{P}$ as

$$
\exists x A+{ }^{t_{1}} E_{1} \cdots+{ }^{t_{n}} E_{n}, \ldots, \exists x A+{ }^{s_{1}} F_{1} \cdots+{ }^{s_{m}} F_{m}, \mathcal{P}^{\prime}
$$

in such a way that: $\operatorname{Sh}(E) \neq \exists x A$ for every expansion tree $E$ in $\mathcal{P}^{\prime}$; there are $i, \ldots, j$ such that $t_{i}=\ldots=t_{n}=t, \ldots, s_{j}=\ldots=s_{m}=t$ and $i^{\prime}<i, \ldots, j^{\prime}<j$ implies $t_{i^{\prime}} \neq t, \ldots, s_{j^{\prime}} \neq t$. Let

$$
\mathcal{Q}=\mathcal{P}^{\prime}, \exists x A+{ }^{t_{1}} E_{1} \cdots+{ }^{t_{i}} E_{i}, \ldots, \exists x A+{ }^{s_{1}} F_{1} \cdots+{ }^{s_{j}} F_{j}, E_{i+1} \ldots, E_{n}, \ldots, F_{j+1}, \ldots, F_{m}
$$

Then $\operatorname{Dp}(\mathcal{P})=\operatorname{Dp}(\mathcal{Q})$, so they are both tautologies. To prove weak regularity of $\mathcal{Q}$, we first observe that every $s \in \operatorname{Br}(\mathcal{Q})$ is either already contained in $\operatorname{Br}(\mathcal{P})$ or $s \in \operatorname{Br}\left(E_{k}\right)$ or $s \in \operatorname{Br}\left(F_{h}\right)$, with $i+1 \leqslant k \leqslant n$ and $j+1 \leqslant h \leqslant m$ and $\exists x A, s$ in $\operatorname{Br}(\mathcal{P})$. Thus the only problematic case is when $b_{1}$ belongs to $\operatorname{Br}(\mathcal{P})$ but not to the branches of the new trees of $\mathcal{Q}$, while, for instance, $b_{2} \in \operatorname{Br}\left(E_{k}\right)$, with $i+1 \leqslant k \leqslant n$. We show that it cannot be the case that $b_{1}=$ $s, \forall y B, B[\alpha / y], s^{\prime}$ and $b_{2}=r, \forall y C, C[\alpha / y], r^{\prime}$ : assume for the sake of contradiction that it is. 
Since $\exists x A, r, \forall y C, C[\alpha / y], r^{\prime} \in \operatorname{Br}(\mathcal{P})$, we have $s=\exists x A, r$, by weak regularity of $\mathcal{P}$. Therefore, $b_{1} \notin \operatorname{Br}\left(\mathcal{P}^{\prime}\right)$, so without loss of generality, say $s=\exists x A, A\left[s_{l} / x\right], s^{\prime \prime}$. But $r=A\left[t_{k} / x\right], r^{\prime \prime}$ and since $s_{l} \neq t=t_{k}$ by construction, we have a contradiction. Furthermore, the orderings of the expansions and cuts of $\mathcal{Q}$ are suborderings of $<_{\mathcal{P}}$, hence also acyclic. Last, no $\forall$-expansion $+^{\beta}$ of $\mathcal{Q}$ can have $\beta$ occur in $A[t / x]$, otherwise either $\beta$ occurs in $t$ or $\beta$ already occurs in $\exists x A$, against the assumptions on minimality of $+^{t}$, in the first case, agains the assumption on $\mathcal{P}$ having the eigenvariable condition, in the second case. Then, by the induction hypothesis we obtain a LKE-proof $\pi$ of $\mathcal{Q}$, from which a proof of $\mathcal{P}$ is obtained by the second $\exists$ rule and a number of applications of the first $\exists$ rule, taking care of the rewriting of $\mathcal{P}$ that we made.

For the case of the minimal node being a $\forall$-expansion, let $\forall x A+{ }^{\alpha} E$ be an expansion tree of $\mathcal{P}$ such that $+^{\alpha}$ is minimal with respect to $<_{\mathcal{P}}$. As we said, $+^{\alpha}$ occurs at top-level. Then $\forall x A, A[\alpha / x], s \in \operatorname{Br}\left(\forall x A+{ }^{\alpha} E\right)$, which, by weak regularity of $\mathcal{P}$, forces every element of $\mathcal{P}$ containing $+^{\alpha}$ to be an expansion tree of the shape $\forall x A+{ }^{\alpha} F$. Then, we can write

$$
\mathcal{P}=\forall x A+{ }^{\alpha} F_{1}, \ldots, \forall x A+{ }^{\alpha} F_{n}, \mathcal{P}^{\prime}
$$

where $+{ }^{\alpha}$ does not occur in $\mathcal{P}^{\prime}$. Now, $\operatorname{Dp}(\mathcal{P})=\operatorname{Dp}\left(F_{1}, \ldots, F_{n}, \mathcal{P}^{\prime}\right)$, so they are both tautologies. To prove weak regularity of $F_{1}, \ldots, F_{n}, \mathcal{P}^{\prime}$, it is enough to note that every $s \in \operatorname{Br}\left(F_{1}, \ldots, F_{n}, \mathcal{P}^{\prime}\right)$ is either already contained in $\operatorname{Br}(\mathcal{P})$ or $s \in F_{k}$, for $1 \leqslant k \leqslant n$ and $\forall x A, s$ is in $\operatorname{Br}(\mathcal{P})$. Thus the only problematic case is when $b_{1}$ belongs to $\operatorname{Br}\left(\mathcal{P}^{\prime}\right)$ but not to the branches of the new trees of $\mathcal{Q}$, while, for instance, $b_{2} \in \operatorname{Br}\left(F_{k}\right)$, with $1 \leqslant k \leqslant n$. We show that it cannot be the case that $b_{1}=s, \forall y B, B[\beta / y], s^{\prime}$ and $b_{2}=r, \forall y C, C[\beta / y], r^{\prime}$ : assume for the sake of contradiction that it is. We first notice that $r=A[\alpha / x], r^{\prime \prime}$. Moreover, since $\forall x A, r, \forall y C, C[\beta / y], r^{\prime} \in \operatorname{Br}(\mathcal{P})$, we have $s=\forall x A, r$, by weak regularity of $\mathcal{P}$. Therefore, $b_{1} \notin \operatorname{Br}\left(\mathcal{P}^{\prime}\right)$ and we have a contradiction. Furthermore, the orderings of the expansions and cuts $F_{1}, \ldots, F_{n}, \mathcal{P}^{\prime}$ are suborderings of $<_{\mathcal{P}}$, hence also acyclic. Last, no $\forall$-expansion $+{ }^{\beta}$ of $F_{1}, \ldots, F_{n}, \mathcal{P}^{\prime}$ can have $\beta$ occur in $A[\alpha / x]$, otherwise either $\beta=\alpha$ or $\beta$ already occurs in $\forall x A$, against the assumptions on $+^{\alpha}$ not occurring in $\mathcal{P}^{\prime}$ or against weak regularity of $\mathcal{P}^{\prime}$, in the first case, against the assumption on $\mathcal{P}$ having the eigenvariable condition, in the second case. Then, by the induction hypothesis we obtain a LKE-proof $\pi$ of $F_{1}, \ldots, F_{n}, \mathcal{P}^{\prime}$, from which a proof of $\mathcal{P}$ is obtained by the $\forall$ rule, because $\alpha$ does not occur in $\operatorname{Sh}(\mathcal{P})$.

\section{Cut-Elimination}

Given any expansion proof $\mathcal{P}$ there is always a cut-free expansion proof of $\operatorname{Sh}(\mathcal{P})$ : by the soundness Theorem 3, transform $\mathcal{P}$ into an LK- proof, then perform Gentzen cut-elimination and obtain a cut-free proof; finally map it back to a cut-free expansion proof by the completeness Theorem 2. Nevertheless, the interest of expansion proofs is that they allow to investigate the combinatorics and the computational meaning of cut-elimination, with the additional advantage of factoring out tedious structural rules such as cut-permutations. In this section we indeed define a natural reduction system for expansion proofs, such that the normal forms are cut-free expansion proofs. We prove weak normalization and discuss the status of other properties such as strong normalization and confluence in comparison to other systems from the literature.

\subsection{Cut-Reduction Steps}

In the following, by a substitution $\sigma$ we mean as usual a finite map from variables to first-order terms, and if $e$ is any syntactic expression, $e \sigma$ denote the expression resulting from $e$ after 
simultaneous replacement of each variable $x$ in the domain of $\sigma$ with $\sigma(x)$. To make sure that the application of a substitution transforms expansion trees into expansion trees we restrict the set of permitted substitutions: a substitution $\sigma$ can only be applied to an expansion tree $E$, if it acts on the eigenvariables of $E$ as a renaming, more precisely: if $+^{\alpha}$ is a $\forall$-expansion of $E$, then $\sigma(\alpha)$ is a variable. Otherwise $\sigma$ would destroy the $\forall$-expansions.

While presenting our cut-reduction steps, we have to take into account weak regularity: cutreduction will duplicate sub-proofs, making it necessary to discuss the renaming of variables, as in the case of the sequent calculus. We will carefully indicate, in the case of a duplication, which subtrees should be subjected to a variable renaming and which variables are to be renamed.

Definition 10 (Cut-Reduction Steps). The cut-reduction steps, relating expansion proofs $\mathcal{Q}, \mathcal{Q}^{\prime}$ and written $\mathcal{Q} \mapsto \mathcal{Q}^{\prime}$, are:

\section{Quantifier Step}

$$
\begin{gathered}
\left\{\exists x A+{ }^{t_{1}} E_{1} \cdots+{ }^{t_{n}} E_{n}, \forall x \bar{A}+{ }^{\alpha_{1}} F_{1}\right\}, \ldots,\left\{\exists x A+{ }^{t_{p}} E_{p} \cdots+{ }^{t_{l}} E_{l}, \forall x \bar{A}+{ }^{\alpha_{q}} F_{q}\right\}, \mathcal{P} \\
\mapsto \\
\left\{E_{1}, F_{1} \eta_{1} \sigma_{1}\right\}, \ldots,\left\{E_{1}, F_{q} \eta_{1} \sigma_{1}\right\}, \ldots,\left\{E_{l}, F_{1} \eta_{l} \sigma_{l}\right\}, \ldots,\left\{E_{l}, F_{q} \eta_{l} \sigma_{l}\right\}, \mathcal{P}, \mathcal{P} \eta_{1} \sigma_{1}, \ldots, \mathcal{P} \eta_{l} \sigma_{l}
\end{gathered}
$$

where: $\sigma_{1}=\left[t_{1} / \alpha_{1} \ldots t_{1} / \alpha_{q}\right], \ldots, \sigma_{l}=\left[t_{l} / \alpha_{1} \ldots t_{l} / \alpha_{q}\right]$; the $\forall$-expansions $+{ }^{\alpha_{1}}, \ldots,+{ }^{\alpha_{q}}$ do not occur in $\mathcal{P}$; no cut in $\mathcal{P}$ has shallow formula $\exists x A ; \eta_{1}, \ldots, \eta_{l}$ are renamings to fresh variables of the eigenvariables $\beta$ of $\mathcal{P}, F_{1}, \ldots, F_{q}$ such that for some $1 \leqslant i \leqslant q$ and occurrence of $+^{\alpha_{i}}$ we have $+{ }^{\alpha_{i}}<\mathcal{P}_{1}+{ }^{\beta}$.

\section{Propositional Step}

$$
\begin{gathered}
\left\{E_{1} \vee F_{1}, E_{1}^{\prime} \wedge F_{1}^{\prime}\right\}, \ldots,\left\{E_{m} \vee F_{m}, E_{m}^{\prime} \wedge F_{m}^{\prime}\right\}, \mathcal{P} \mapsto\left\{E_{1}, E_{1}^{\prime}\right\},\left\{F_{1}, F_{1}^{\prime}\right\}, \ldots,\left\{E_{m}, E_{m}^{\prime}\right\},\left\{F_{m}, F_{m}^{\prime}\right\}, \mathcal{P} \\
\text { where } \operatorname{Sh}\left(E_{1} \vee F_{1}\right)=\cdots=\operatorname{Sh}\left(E_{m} \vee F_{m}\right) \text { and for every } C \text { in } \mathcal{P}, \operatorname{Sh}(C) \neq \operatorname{Sh}\left(E_{1} \vee F_{1}\right) . \\
\text { Atomic Step } \\
\{A, \bar{A}\}, \mathcal{P} \mapsto \mathcal{P} \text { for an atom } A .
\end{gathered}
$$

These reduction rules are very natural: atomic cuts are simply removed and propositional cuts are decomposed. The reduction of a quantified cut is, when thinking about cut-elimination in the sequent calculus, intuitively appealing: existential cuts are replaced by cuts on a disjunction of the instances. The fact that at least one of these rules that can be applied to any expansion proof containing cuts will be proved in Theorem 6 .

One may think that the quantifier reduction rule already incorporates a reduction strategy, because several cuts are reduced in parallel. However, a strategy implies a choice and there is no real choice here: when the main eigenvariable occurs in other cuts, all these cuts have to be regarded as linked together, otherwise reducing one of them would destroy the soundness of the others. Moreover, all the cuts with the same shallow formula must be reduced, otherwise weak regularity would not be preserved.

The reason why only the eigenvariables greater than some $\alpha_{i}$ are renamed is that these are the variable indirectly affected by the substitutions $\left[t_{i} / \alpha\right]$. Semantically, the witnesses that these variables represent are influenced by the substitutions, so for each of them a new collection of eigenvariables is created.

One surprising aspect of the quantifier-reduction rule is the presence of $\mathcal{P}$, without a substitution applied, on the right-hand side of the rule: in general, $\mathcal{P}$ will contain $\alpha$, and one would expect that occurrences of $\alpha$ are redundant (since $\alpha$ is "eliminated" by the rule). The reason why this 
occurrence of $\mathcal{P}$ must be present is that $\alpha$ is not, in fact, eliminated since some $t_{i}$ might contain it. This situation occurs, for example, when translating from a regular LK-proof where an $\exists$-quantifier may be instantiated by any term, and we happen to choose an eigenvariable from a different branch of the proof. In the sequent calculus, this situation can in principle be avoided by using a different witness for the $\exists$-quantifier, but realizing such a renaming in expansion proofs is technically non-trivial due to the global nature of eigenvariables. For simplicity of exposition, we therefore allow this somewhat unnatural situation and leave a more detailed analysis for future work. Note that this $n+1$-st copy is reminiscent of the duplication behavior of the $\varepsilon$-calculus [17], see [21] for a contemporary exposition in English.

Remark 1 (On Bridges). We note that this phenomenon also occurs in the proof forests of [13], where it is an example of bridge. Bridges, when ignored, can generate cycles in the dependency relation $<_{\mathcal{Q}}$. In [13], they are addressed with a pruning reduction that eliminates them and the weak normalization proof of that system depends on this pruning. In our setting, we do not need additional machinery for proving weak normalization (see Section 4.3). The reason is our renaming policy: while in [13] every occurrence of every variable above $\alpha$ in the dependency relation $<_{\mathcal{Q}}$ is renamed, in our case only some of those occurrences are renamed, namely those which are not in $t_{1}, \ldots, t_{n}$ or in $E_{1}, \ldots, E_{n}$. In such a way, bridges are broken by our cutreduction step, so that cycles in the dependency relation cannot be generated. Furthermore, the counterexample to strong normalization from [13] also contains a bridge; we investigate ( $a$ translation of) this counterexample in Section 4.4 and find that it is not a counterexample in our setting for the reasons explained.

Example 2. We now consider an example of cut reduction steps, in particular when an eigenvariable $\gamma$ occurs more than once.

$$
\begin{gathered}
\left\{\forall x \exists y P(x, y)+{ }^{\alpha} \exists y P(\alpha, y)+{ }^{f(\alpha, \beta)} P(\alpha, f(\alpha, \beta)), \exists x \forall y \bar{P}(x, y)+{ }^{q} \forall y \bar{P}(q, y)+{ }^{\gamma} \bar{P}(q, \gamma)\right\}, \\
\left\{\forall x \exists y P(x, y)+{ }^{\beta} \exists y P(\beta, y)+{ }^{g(\alpha, \beta)} P(\beta, g(\alpha, \beta)), \exists x \forall y \bar{P}(x, y)+{ }^{q} \forall y \bar{P}(q, y)+{ }^{\gamma} \bar{P}(q, \gamma)\right\} \\
\mapsto \\
\left\{\exists y P(q, y)+{ }^{f(q, q)} P(q, f(q, q)), \forall y \bar{P}(q, y)+{ }^{\gamma} \bar{P}(q, \gamma)\right\}, \\
\left\{\exists y P(q, y)+{ }^{g(q, q)} P(q, g(q, q)), \forall y \bar{P}(q, y)+{ }^{\gamma} \bar{P}(q, \gamma)\right\}, \\
\left\{\exists y P(q, y)+{ }^{f(q, q)} P(q, f(q, q)), \forall y \bar{P}(q, y)+{ }^{\gamma} \bar{P}(q, \gamma)\right\}, \\
\left\{\exists y P(q, y)+{ }^{g(q, q)} P(q, g(q, q)), \forall y \bar{P}(q, y)+{ }^{\gamma} \bar{P}(q, \gamma)\right\},
\end{gathered}
$$

We see above two identical pairs of cuts, due to the first expansion containing two times the same tree. Reducing this last expansion, we obtain 8 occurrences of $\{P(q, f(q, q)), \bar{P}(q, f(q, q)\}$ and $\{P(p, f(p, p)), \bar{P}(p, f(p, p)\}$. $\operatorname{Br}(\cdot)$.

A simple property we are going to need is that substitution commutes with $\mathrm{Dp}(\cdot), \mathrm{Sh}(\cdot)$ and 
Lemma 4. For every substitution $\sigma$,

$$
\begin{gathered}
\operatorname{Sh}(E \sigma)=\operatorname{Sh}(E) \sigma \\
\operatorname{Dp}(E \sigma)=\operatorname{Dp}(E) \sigma \\
\operatorname{Br}(E \sigma)=\{s \sigma \mid s \in \operatorname{Br}(E)\}
\end{gathered}
$$

Proof. By a straightforward induction on $E$.

We now prove that the cut-reduction relation is sound.

Lemma 5 (Soundness of Cut-Reduction). If $\mathcal{P}_{1} \mapsto \mathcal{P}_{2}$ and $\mathcal{P}_{1}$ is an expansion proof, then $\mathcal{P}_{2}$ is an expansion proof. Furthermore, $\operatorname{Sh}\left(\mathcal{P}_{1}\right)=\operatorname{Sh}\left(\mathcal{P}_{2}\right)$.

Proof. We only give the proof for the quantifier cut-reduction step; the proof for the other reduction steps is analogous and simpler. Let $\sigma_{1}, \ldots, \sigma_{l}$ be respectively the substitutions $\left[t_{1} / \alpha_{1} \ldots t_{1} / \alpha_{q}\right], \ldots,\left[t_{l} / \alpha_{1} \ldots t_{l} / \alpha_{q}\right]$ and assume

$$
\begin{gathered}
\mathcal{P}_{1}=\left\{\exists x A+{ }^{t_{1}} E_{1} \cdots+{ }^{t_{n}} E_{n}, \forall x \bar{A}+{ }^{\alpha_{1}} F_{1}\right\}, \ldots,\left\{\exists x A+{ }^{t_{p}} E_{p} \cdots+{ }^{t_{l}} E_{l}, \forall x \bar{A}+{ }^{\alpha_{q}} F_{q}\right\}, \mathcal{P} \\
\mapsto \\
\left\{E_{1}, F_{1} \eta_{1} \sigma_{1}\right\}, \ldots,\left\{E_{1}, F_{q} \eta_{1} \sigma_{1}\right\}, \ldots,\left\{E_{l}, F_{1} \eta_{l} \sigma_{l}\right\}, \ldots,\left\{E_{l}, F_{q} \eta_{l} \sigma_{l}\right\}, \mathcal{P}, \mathcal{P} \eta_{1} \sigma_{1}, \ldots, \mathcal{P} \eta_{l} \sigma_{l} \\
=\mathcal{Q}
\end{gathered}
$$

where the $\forall$-expansions $+{ }^{\alpha_{1}}, \ldots,+{ }^{\alpha_{q}}$ do not occur in $\mathcal{P}$, no cut in $\mathcal{P}$ has shallow formula $\exists x A$ and $\eta_{1}, \ldots, \eta_{l}$ are renamings to fresh variables of the eigenvariables $\beta$ of $\mathcal{P}, F_{1}, \ldots, F_{q}$ such that for some $1 \leqslant i \leqslant q$ and occurrence of $+{ }^{\alpha_{i}}$ we have $+{ }^{\alpha_{i}}<\mathcal{P}_{1}+{ }^{\beta}$. We observe that no $\alpha_{k}$ is in the domain of any $\eta_{j}$, otherwise for some $i$ we would have $+{ }^{\alpha_{i}}<\mathcal{P}_{1}+{ }^{\alpha_{k}}$, which is only possible when $+{ }^{\alpha_{i}}<_{\mathcal{P}_{1}}+{ }^{\gamma}<_{\mathcal{P}_{1}} C_{k}$ where $\gamma$ is an eigenvariable and $C_{k}$ is the $k$-th of the displayed cuts of $\mathcal{P}_{1}$. But then $\gamma$ would occur in $\exists x A$, hence also $+{ }^{\alpha_{i}}<\mathcal{P}_{1}+{ }^{\alpha_{i}}$, which contradicts the acyclicity of $<_{\mathcal{P}_{1}}$. We now show that no variable $\gamma$ in the domain of any $\eta_{k}$ can occur in any $\bar{A}\left[\alpha_{j} / x\right]=\operatorname{Sh}\left(F_{j}\right)$ : assume by contradiction that $+{ }^{\alpha_{i}}<_{\mathcal{P}_{1}}+{ }^{\gamma}$ and $\gamma$ occurs in $\bar{A}\left[\alpha_{j} / x\right]$. Then, letting $C_{i}$ be the $i$-th of the displayed cuts of $\mathcal{P}_{1}$, we immediately have by Definition 5 the contradiction $C_{i}<\mathcal{P}_{1}+{ }^{\alpha_{i}}<\mathcal{P}_{1}+{ }^{\gamma}<\mathcal{P}_{1} C_{i}$, the last relation due to $\gamma \neq \alpha_{j}$ as shown above, thus $\gamma$ occurring in $\forall x \bar{A}$ and thus in $\operatorname{Sh}(C)=\exists x A$. Therefore, by Lemma 4 , for every $1 \leqslant h \leqslant q$, we have $\operatorname{Sh}\left(F_{h} \eta_{i} \sigma_{i}\right)=\operatorname{Sh}\left(F_{h}\right) \eta_{i} \sigma_{i}=\operatorname{Sh}\left(F_{h}\right) \sigma_{i}=\bar{A}\left[t_{i} / x\right]$, the last equality due to $\alpha_{j}$ for $j \neq i$ not occurring in $\bar{A}$, otherwise $+{ }^{\alpha_{i}}<\mathcal{P}_{1}+{ }^{\alpha_{j}}$. We have thus shown that the displayed cuts of $\mathcal{Q}$ are indeed between dual formulas.

We now prove several properties, namely that $\mathcal{Q}$ is weakly regular, $\operatorname{Dp}(Q)$ is valid, $<_{\mathcal{Q}}$ is acyclic and $\operatorname{Sh}(\mathcal{Q})$ does not contain eigenvariables, which means that $\mathcal{Q}$ is an expansion proof.

Weak Regularity. Letting $\eta_{0}$ and $\sigma_{0}$ be the empty substitution, by Lemma 4 any branch in $\operatorname{Br}(\mathcal{Q})$ is of the form $p \eta_{i} \sigma_{i}$, where $i \in\{0,1, \ldots, n\}$ and

$$
p \in \mathrm{B}:=\operatorname{Br}(\mathcal{P}) \cup \operatorname{Br}\left(E_{1}\right) \cup \ldots \cup \operatorname{Br}\left(E_{l}\right) \cup \ldots \cup \operatorname{Br}\left(F_{1}\right) \cup \ldots \cup \operatorname{Br}\left(F_{q}\right)
$$

Thus, for each $p \in \mathrm{B}$, either $p \in \operatorname{Br}(\mathcal{P})$ or $\forall x \bar{A}, p \in \operatorname{Br}\left(\mathcal{P}_{1}\right)$ or $\exists x A, p \in \operatorname{Br}\left(\mathcal{P}_{1}\right)$. Assume

$$
\begin{gathered}
s \eta_{i} \sigma_{i}, \forall x B \eta_{i} \sigma_{i}, B[\beta / x] \eta_{i} \sigma_{i}, s^{\prime} \eta_{i} \sigma_{i} \in \operatorname{Br}(S) \\
r \eta_{j} \sigma_{j}, \forall x C \eta_{j} \sigma_{j}, C[\delta / x] \eta_{j} \sigma_{j}, r^{\prime} \eta_{j} \sigma_{j} \in \operatorname{Br}(R)
\end{gathered}
$$

with $S$ and $R$ in $\mathcal{Q}$. Let $\bar{s}=s, \forall x B, B[\beta / x], s^{\prime}$ and $\bar{r}=r, \forall x C, C[\delta / x], r^{\prime}$. We first rule out that either $\bar{s} \in \mathrm{B}$ and $J x A, \bar{r} \in \mathrm{B}$ or $J x A, \bar{s} \in \mathrm{B}$ and $\bar{r} \in \mathrm{B}$, with $J \in\{\forall, \exists\}$. Indeed, since in the 
first case $J x A, \bar{r} \in \mathrm{B}$ and in the second case $J x A, \bar{s} \in \mathrm{B}$ belong to the branches of one of the displayed cuts of $\mathcal{P}_{1}$, by weak regularity of $\mathcal{P}_{1}$ we would have that $\bar{s}$ or $\bar{r}$ belong to the branches of a cut in $\mathcal{P}$ with shallow formula $\exists x A$, contrary to our assumptions.

Now there are two cases.

- If $\beta$ is in the domain of $\eta_{i}$, then it is also in the domain of $\eta_{j}$ and

$$
\begin{aligned}
& B[\beta / x] \eta_{i} \sigma_{i}=B \eta_{i} \sigma_{i}\left[\eta_{i}(\beta) / x\right] \\
& C[\delta / x] \eta_{j} \sigma_{j}=C \eta_{j} \sigma_{j}\left[\eta_{j}(\delta) / x\right]
\end{aligned}
$$

By Definition 6 of weak regularity, we suppose $\eta_{i}(\beta)=\eta_{j}(\delta)$ and then we have to check that: i) $S$ and $R$ are both trees or cuts; ii)

$$
s \eta_{i} \sigma_{i}, \forall x B \eta_{i} \sigma_{i}=r \eta_{j} \sigma_{j}, \forall x C \eta_{j} \sigma_{j}
$$

First, we have $i=j$ and $\beta=\delta$, because $\eta_{i}$ and $\eta_{j}$ are both injective and have disjoint domains by the freshness assumption on the renamings. Now, since either $\bar{s} \in \mathrm{B}$ and $\bar{r} \in \mathrm{B}$ or $J_{1} x A, \bar{s} \in \mathrm{B}$ and $J_{2} x A, \bar{r} \in \mathrm{B}$, by weak regularity of $\mathcal{P}_{1}$ we infer i) and

$$
s, \forall x B=r, \forall x C
$$

thus ii) also follows.

- If $\beta$ is not in the domain of $\eta_{i}$, then it is not in the domain of $\eta_{j}$ either and

$$
\begin{aligned}
& B[\beta / x] \eta_{i} \sigma_{i}=B \eta_{i} \sigma_{i}[\beta / x] \\
& C[\delta / x] \eta_{j} \sigma_{j}=C \eta_{j} \sigma_{j}[\delta / x]
\end{aligned}
$$

since the $\forall$-expansions $+{ }^{\alpha_{i}},+{ }^{\alpha_{j}}$ by assumption do not occur in $\mathcal{P}$ and thus in $\mathcal{Q}$. By Definition 6 of weak regularity, we suppose $\beta=\delta$ and then we have to check that: i) $S$ and $R$ are both trees or cuts; ii)

$$
s \eta_{i} \sigma_{i}, \forall x B \eta_{i} \sigma_{i}=r \eta_{j} \sigma_{j}, \forall x C \eta_{j} \sigma_{j}
$$

Now, since either $\bar{s} \in \mathrm{B}$ and $\bar{r} \in \mathrm{B}$ or $J_{1} x A, \bar{s} \in \mathrm{B}$ and $J_{2} x A, \bar{r} \in \mathrm{B}$, by weak regularity of $\mathcal{P}_{1}$ we infer i) and

$$
s, \forall x B=r, \forall x C
$$

If $i=j$, we are done. So assume $i \neq j$. We want to show that no variable in the domain of $\eta_{i} \sigma_{i}$ or of $\eta_{j} \sigma_{j}$ appears in $s, \forall x B$ or $s, \forall x C$, so that we are done. Indeed, if there was such a variable $\gamma$, then there would be either some expansion $v$ containing $\gamma$ or some cut $v$ with $\operatorname{Sh}(v)$ containing $\gamma$ such that $v$ dominates $+{ }^{\beta}$ in $\mathcal{P}$ and thus $v<\mathcal{P}_{1}+{ }^{\beta}$. By choice of $\eta_{i}, \eta_{j}$, we have $+{ }^{\alpha}{ }^{\alpha}<\mathcal{P}_{1}+{ }^{\gamma}$, where $k \in\{i, j\}$ and moreover $+^{\gamma}<_{\mathcal{P}_{1}} v$; thus putting the three together, $+{ }^{\alpha}{ }^{\alpha}<\mathcal{P}_{1}+{ }^{\gamma}<\mathcal{P}_{1} v<\mathcal{P}_{1}+{ }^{\beta}$. Therefore, $\beta$ is in the domain of $\eta_{k}$, though we were assuming it is not.

Validity. The formula

$$
\operatorname{Dp}\left(\mathcal{P}_{1}\right)=\left(\bigvee_{i=1}^{n} \operatorname{Dp}\left(E_{i}\right) \wedge \operatorname{Dp}\left(F_{1}\right)\right) \vee \ldots \vee\left(\bigvee_{i=p}^{l} \operatorname{Dp}\left(E_{i}\right) \wedge \operatorname{Dp}\left(F_{q}\right)\right) \vee \operatorname{Dp}(\mathcal{P})
$$


by assumption is valid and logically implies the formulas

$$
\begin{gathered}
\text { (1) } \bigvee_{i=1}^{n} \operatorname{Dp}\left(E_{i}\right) \vee \ldots \vee \bigvee_{i=p}^{l} \operatorname{Dp}\left(E_{i}\right) \vee \operatorname{Dp}(\mathcal{P}) \\
\operatorname{Dp}\left(F_{1}\right) \vee \ldots \vee \operatorname{Dp}\left(F_{q}\right) \vee \operatorname{Dp}(\mathcal{P})
\end{gathered}
$$

which then are valid too. Therefore, also the formula

$$
\text { (2) } \bigwedge_{i=1}^{l}\left(\operatorname{Dp}\left(F_{1}\right) \eta_{i} \sigma_{i} \vee \ldots \vee \operatorname{Dp}\left(F_{q}\right) \eta_{i} \sigma_{i} \vee \operatorname{Dp}(\mathcal{P}) \eta_{i} \sigma_{i}\right)
$$

is valid. We have that $\operatorname{Dp}(\mathcal{Q})$ is equal to the formula

$$
\begin{gathered}
\left(\operatorname{Dp}\left(E_{1}\right) \wedge \operatorname{Dp}\left(F_{1}\right) \eta_{1} \sigma_{1}\right) \vee \ldots \vee\left(\operatorname{Dp}\left(E_{1}\right) \wedge \operatorname{Dp}\left(F_{q}\right) \eta_{1} \sigma_{1}\right) \vee \\
\ldots \vee\left(\operatorname{Dp}\left(E_{l}\right) \wedge \operatorname{Dp}\left(F_{1}\right) \eta_{l} \sigma_{l}\right) \vee \ldots \vee\left(\operatorname{Dp}\left(E_{l}\right) \wedge \operatorname{Dp}\left(F_{q}\right) \eta_{l} \sigma_{l}\right) \vee \\
\operatorname{Dp}(\mathcal{P}) \vee \bigvee_{i=1}^{l} \operatorname{Dp}\left(\mathcal{P} \eta_{i} \sigma_{i}\right)
\end{gathered}
$$

Now, fix any propositional truth assignment. Assume $\operatorname{Dp}(\mathcal{P}) \vee \bigvee_{i=1}^{l} \operatorname{Dp}\left(\mathcal{P} \eta_{i} \sigma_{i}\right)$ is false under this assignment. By $(1)$, we can assume $\operatorname{Dp}\left(E_{1}\right)$ is true (if one among $\operatorname{Dp}\left(E_{2}\right), \ldots, \operatorname{Dp}\left(E_{l}\right)$ is true, the reasoning is symmetric). Indeed, by $(2)$, the formula $\operatorname{Dp}\left(F_{1}\right) \eta_{1} \sigma_{1} \vee \ldots \vee \operatorname{Dp}\left(F_{q}\right) \eta_{1} \sigma_{1}$ is true, thus the formula

$$
\left(\mathrm{Dp}\left(E_{1}\right) \wedge \mathrm{Dp}\left(F_{1}\right) \eta_{1} \sigma_{1}\right) \vee \ldots \vee\left(\mathrm{Dp}\left(E_{1}\right) \wedge \mathrm{Dp}\left(F_{q}\right) \eta_{1} \sigma_{1}\right)
$$

is true and finally $\mathcal{Q}$ is true.

Acyclicity. We show that acyclicity of $<_{\mathcal{P}_{1}}$ implies acyclicity of $<_{\mathcal{Q}}$. Our strategy is to map any purported cycle of $\mathcal{Q}$ into a cycle of $\mathcal{P}_{1}$.

The first difficulty we are going to face is that the expansions of $\mathcal{Q}$ are not well-behaved copies of expansions of $\mathcal{P}_{1}$, because of the substitutions $\sigma_{i}$. For example, an $\exists$-expansion $+{ }^{u}$ of $\mathcal{P}$ could contain some $\alpha_{j}$, so that we would find $+^{u\left[t_{i} / \alpha_{j}\right]}$ in $\mathcal{Q}$. The trouble is that $+^{u\left[t_{i} / \alpha_{j}\right]}$ could contain variables that were not in $u$, thus there could be $\forall$-expansions $+{ }^{\beta}$ such that $+^{\beta}<_{\mathcal{Q}}+{ }^{u\left[t_{i} / \alpha\right]}$ but it is not the case that $+{ }^{\beta}<_{\mathcal{P}_{1}}+{ }^{u}$. This means that the relation $<_{\mathcal{Q}}$ presents entirely new paths that were not in $\mathcal{P}_{1}$. We might encounter paths in $\mathcal{Q}$ that a priori could not be mapped back to paths in $\mathcal{P}_{1}$ : for example, a path featuring copies of old expansions suddenly followed by new ill-behaved expansion like $+{ }^{u\left[t_{i} / \alpha_{j}\right]}$. However, we shall take care of this issue in a next Lemma, explaining that such paths cannot end up in a cycle, because whatever path enters the "renamed zone" is stuck in it. The basic intuition is that since $u$ contains $\alpha_{j}$, everything in relation $<_{\mathcal{Q}}$ with $+{ }^{u\left[t_{i} / \alpha_{j}\right]}$ is under the scope of the renaming $\eta_{j}$ and all the renamed $\forall$-expansion have a renamed variable that can "jump" only in the renamed zone.

The second difficulty we are going to encounter is that one of the new displayed cuts $\left\{E_{k}, F_{h} \eta_{k} \sigma_{k}\right\}$ of $\mathcal{Q}$ may have no direct correspondents in $\mathcal{P}_{1}$. Again, the problem is that the term $t_{k}$ introduced by the substitution $\sigma_{k}$ could add some variable $\beta$ to the shallow formula $A\left[t_{k} / x\right]$ of the new cut which is not in the shallow formula $\exists x A$ of the original cuts. In this case, some $\forall$-expansion $+^{\beta}$ would be in relation with the new cut $\left\{E_{k}, F_{h} \eta_{k} \sigma_{k}\right\}$ of $\mathcal{Q}$, but with none of the old ones of $\mathcal{P}_{1}$. In this case the new cut will be mapped to $+{ }^{t_{k}}$, in all others to the old cut $\left\{\exists x A+{ }^{t_{i}} E_{i} \cdots+{ }^{t_{j}} E_{j}, \forall x \bar{A}+{ }^{\alpha_{s}} F_{s}\right\}$ such that $i \leqslant k \leqslant j$. But when the new cut is mapped to 
$+{ }^{t_{k}}$, one has to make sure that the new cut is not in relation in the cycle with some expansion in $F_{h} \eta_{k} \sigma_{k}$, otherwise the mapping we wish to build would fail. Luckily, the old expansion $+{ }^{\beta}$ of $\mathcal{P}_{1}$ ( $\beta$ occurs in $t_{k}$ ) cannot jump in the "renamed zone" if it is part of a cycle. The "renamed zone" argument settles the issue also when $s \neq h$, which would cause our mapping to fail in the way we have just discussed.

Let us now work out the formal details. Consider any cycle

$$
v_{1}<_{\mathcal{Q}} v_{2}<_{\mathcal{Q}} \ldots<_{\mathcal{Q}} v_{m}<_{\mathcal{Q}} v_{1}
$$

in $\mathcal{Q}$. Then each $v_{i}$ is either of the form $w_{i} \eta_{k} \sigma_{k}$ for some $w_{i}$ which is an old expansion or cut belonging to $\mathcal{P}_{1}$ or $v_{i}$ is one of the new displayed cuts $\left\{E_{k}, F_{h} \eta_{k} \sigma_{k}\right\}$ of $\mathcal{Q}$; in this second case, we define $w_{i}$ to be the displayed cut $\left\{\exists x A+{ }^{t_{i}} E_{i} \cdots+{ }^{t_{j}} E_{j}, \forall x \bar{A}+{ }^{\alpha_{s}} F_{s}\right\}$ of $\mathcal{P}_{1}$ such that $i \leqslant k \leqslant j$, if the eigenvariable of the $\forall$-expansion $v_{i-1(\bmod m)}$ does not occur in $t_{k}$, or to be the displayed occurrence of $+{ }^{t_{k}}$ in $\mathcal{P}_{1}$ otherwise. We want to show that

$$
w_{1}<\mathcal{P}_{1} w_{2}<\mathcal{P}_{1} \ldots<\mathcal{P}_{1} w_{m}<\mathcal{P}_{1} w_{1}
$$

First of all we need the following:

"Renamed Zone" Lemma. Suppose $\alpha \in\left\{\alpha_{1}, \ldots, \alpha_{q}\right\}$. If there are $k$ and $j>0$ such that $+{ }^{\alpha}<_{\mathcal{P}_{1}} w_{k}$ and $v_{k}$ occurs in $\mathcal{P} \eta_{j} \sigma_{j}$ or $F_{h} \eta_{j} \sigma_{j}$, then for all $i,{ }^{\alpha}{ }^{\alpha} \mathcal{P}_{1} w_{i}$ and $v_{i}$ occurs in $\mathcal{P} \eta_{j} \sigma_{j}$ or $F_{h} \eta_{j} \sigma_{j}$.

Proof of the Lemma. Since we are dealing with a cycle, we may assume without loss of generality that $k=1$. We proceed by induction on $i$, the case $i=1$ being already settled. Suppose by induction hypothesis that $+^{\alpha}<_{\mathcal{P}_{1}} w_{i}$ and $v_{i}$ occurs in $\mathcal{P} \eta_{j} \sigma_{j}$ or $F_{h} \eta_{j} \sigma_{j}$ and thus $w_{i}$ occurs in $\mathcal{P}$ or $F_{h}$. If $v_{i}$ dominates $v_{i+1}$, then $w_{i}$ dominates $w_{i+1}$ and thus $+{ }^{\alpha}<_{\mathcal{P}_{1}} w_{i+1}$ and $v_{i+1}$ occurs in $\mathcal{P} \eta_{j} \sigma_{j}$ or $F_{h} \eta_{j} \sigma_{j}$. Suppose then $v_{i}$ is a $\forall$-expansion, thus also $w_{i}=+^{\beta}$ is a $\forall$-expansion and the eigenvariable of $v_{i}$ occurs in $v_{i+1}$ or $\operatorname{Sh}\left(v_{i+1}\right)$. Since $+{ }^{\alpha}<\mathcal{P}_{1}+{ }^{\beta}$ and $v_{i} \in \mathcal{P} \eta_{j} \sigma_{j}$ or $F_{h} \eta_{j} \sigma_{j}$, we have $v_{i}=+^{\eta_{j}(\beta)}$. Now, $\eta_{j}(\beta)$ is fresh and as it occurs in $v_{i+1}$ or $\operatorname{Sh}\left(v_{i+1}\right)$, we have that $v_{i+1}$ must occur in $\mathcal{P} \eta_{j} \sigma_{j}$ or $F_{h} \eta_{j} \sigma_{j}$. Moreover, $v_{i+1}$ cannot be one of the new cuts of $\mathcal{Q}$, because $\operatorname{Sh}(w)$ would not contain $\eta_{j}(\beta)$. Therefore, $v_{i+1}=w_{i+1} \eta_{j} \sigma_{j}$; since $\eta_{j}(\beta)$ occurs in $v_{i+1}$ and is fresh, it also occurs in $w_{i+1} \eta_{j}$ and thus $\beta$ must occur in $w_{i+1}$ or $\operatorname{Sh}\left(w_{i+1}\right)$, so that $+{ }^{\alpha}<_{\mathcal{P}_{1}}+{ }^{\beta}<_{\mathcal{P}_{1}} w_{i+1}$, which ends the proof of the Lemma.

We now prove, by induction on $i$, that for every $i, w_{i}<_{\mathcal{P}_{1}} w_{i+1}$ (in the following the indexes $i$ of $w_{i}$ and $v_{i}$ will be taken modulo $m$ ).

If $v_{i}$ dominates $v_{i+1}$, there are three possibilities:

- $w_{i}$ is an old cut or expansion of $\mathcal{P}_{1}$ and $v_{i}=w_{i} \eta_{k} \sigma_{k}$. Then also $v_{i+1}=w_{i+1} \eta_{k} \sigma_{k}$, where $w_{i+1}$ is an old expansion of $\mathcal{P}_{1}$, therefore also $w_{i}$ dominates $w_{i+1}$ and we get $w_{i}<_{\mathcal{P}_{1}} w_{i+1}$.

- $w_{i}$ is the displayed cut $\left\{\exists x A+{ }^{t_{i}} E_{i} \cdots+{ }^{t_{j}} E_{j}, \forall x \bar{A}+{ }^{\alpha_{s}} F_{s}\right\}$ of $\mathcal{P}_{1}$ such that $i \leqslant k \leqslant j$ and $v_{i}=\left\{E_{k}, F_{h} \eta_{k} \sigma_{k}\right\}$. Since $v_{i}$ dominates $v_{i+1}$, either $v_{i+1}$ occurs in $E_{k}$ or $v_{i+1}$ occurs in $F_{h} \eta_{k} \sigma_{k}$. In the first case, $v_{i+1}=w_{i+1}$, thus $w_{i}<_{\mathcal{P}_{1}} w_{i+1}$. The second case is not possible: if $v_{i+1}$ occurred in $F_{h} \eta_{k} \sigma_{k}$, then $v_{i+1}=w_{i+1} \eta_{k} \sigma_{k}$ with $w_{i+1}$ occurring in $F_{h}$. Therefore, $+{ }^{\alpha_{h}}<\mathcal{P}_{1} w_{i+1}$ and by the "Renamed Zone" Lemma, $+{ }^{\alpha_{h}}<_{\mathcal{P}_{1}} w_{i-1}$ and $v_{i-1}$ occurs in $\mathcal{P} \eta_{k} \sigma_{k}$ or $F_{h} \eta_{k} \sigma_{k}$. Moreover, $v_{i-1}=+^{\beta}$, with $\beta$ occurring in $\bar{A}\left[t_{k} / x\right]$. Thus $\beta$ must be a variable of $\mathcal{P}_{1}$ and $+{ }^{\alpha}{ }^{\alpha}<\mathcal{P}_{1} w_{i-1}=+^{\beta}$. But then $+{ }^{\beta}$ cannot occur in $\mathcal{P} \eta_{k} \sigma_{k}$ or $F_{h} \eta_{k} \sigma_{k}$, because $\beta$ is in the domain, but not in the range, of $\eta_{k}$ : contradiction. 
- $w_{i}=+^{t_{k}}, v_{i}=\left\{E_{k}, F_{h} \eta_{k} \sigma_{k}\right\}$. Since $v_{i}$ dominates $v_{i+1}$, either $v_{i+1}$ occurs in $E_{k}$ or $v_{i+1}$ occurs in $F_{h} \eta_{k} \sigma_{k}$. The second case is excluded as before. In the first case, $v_{i+1}=w_{i+1}$, therefore $w_{i}<_{\mathcal{P}_{1}} w_{i+1}$.

Suppose then $v_{i}$ is an $\forall$-expansion and thus $w_{i}=+^{\gamma}$ is a $\forall$-expansion as well. We have two cases.

1. $v_{i}=+^{\eta_{j}(\gamma)}$. Now, as $v_{i}<_{\mathcal{Q}} v_{i+1}$, we know that $\eta_{j}(\gamma)$ occurs in $v_{i+1}$ or in $\operatorname{Sh}\left(v_{i+1}\right)$. Moreover, $v_{i+1}$ cannot be one of the new displayed cuts of $\mathcal{Q}$, because $\operatorname{Sh}\left(v_{i+1}\right)=A\left[t_{k} / x\right]$ or $\operatorname{Sh}\left(v_{i+1}\right)=\bar{A}\left[t_{k} / x\right]$ and since $\eta_{j}(\gamma)$ is fresh, it cannot occur in those formulas.

Thus $v_{i+1}$ is the result of a substitution in an old cut (resp. expansion) $w_{i+1}, \operatorname{so} \operatorname{Sh}\left(v_{i+1}\right)=$ $\operatorname{Sh}\left(w_{i+1}\right) \eta_{k}\left[t_{k} / \alpha\right]$ (resp. $\left.v_{i+1}=w_{i+1} \eta_{k}\left[t_{k} / \alpha\right]\right)$. Since $\eta_{j}(\gamma)$ is fresh, it cannot occur in $t_{k}$, therefore $j=k$ and $\gamma$ must occur also in $\operatorname{Sh}\left(w_{i+1}\right)$ (resp. $w_{i+1}$ ) and thus $w_{i}=+^{\gamma}<\mathcal{P}_{1}$ $w_{i+1}$.

2. $v_{i}=+^{\gamma}$ and $\gamma$ occurs in $v_{i+1}$ or in $\operatorname{Sh}\left(v_{i+1}\right)$. Now we are left with two possibilities.

- $v_{i+1}=w_{i+1} \eta_{k} \sigma_{k}$. If $k=0$, then $v_{i+1}=w_{i+1}$ and we are done. Moreover, if no $\alpha \in\left\{\alpha_{1}, \ldots, \alpha_{q}\right\}$ occurs in $w_{i+1}$ or $\gamma$ does not occur in $t_{k}$, then $\gamma$ occurs in $w_{i+1}$, which means $w_{i}<\mathcal{P}_{1} w_{i+1}$. Suppose thus by contradiction that they do. Then $+{ }^{\alpha}<_{\mathcal{P}_{1}} w_{i+1}$ and $v_{i+1}$ occurs in $\mathcal{P} \eta_{k} \sigma_{k}$ or $F_{h} \eta_{k} \sigma_{k}$. By the "Renamed Zone" Lemma, we conclude that $+^{\alpha}<_{\mathcal{P}_{1}}+{ }^{\gamma}$ and $+^{\gamma}$ occurs in $\mathcal{P} \eta_{k} \sigma_{k}$ or $F_{h} \eta_{k} \sigma_{k}$ : but then $\gamma$ is in the domain of $\eta_{k}$, whereas $+^{\gamma}$ occurs in $\mathcal{P}$ or $F_{h}$, contradiction.

- $v_{i+1}=\left\{E_{k}, F_{h} \eta_{k} \sigma_{k}\right\}$ is one of the new cuts of $\mathcal{Q}$. Since $v_{i}<_{\mathcal{Q}} v_{i+1}, \gamma$ occurs in $\operatorname{Sh}\left(v_{i+1}\right)=A\left[t_{k} / x\right]$. If $\gamma$ does not occur in $t_{k}$, then by definition of $w_{i+1}, \gamma$ occurs in

$$
\exists x A=\operatorname{Sh}\left(\left\{\exists x A+{ }^{t_{i}} E_{i} \cdots+{ }^{t_{j}} E_{j}, \forall x \bar{A}+{ }^{\alpha} F_{s}\right\}\right)=\operatorname{Sh}\left(w_{i+1}\right)
$$

with $i \leqslant k \leqslant j$, so $w_{i}<_{\mathcal{P}} w_{i+1}$. If $\gamma$ does occur in $t_{k}$, then $w_{i+1}=+{ }^{t_{k}}$, so $w_{i}<_{\mathcal{Q}} w_{i+1}$.

Eigenvariable condition. The fact that the eigenvariable of every $\forall$-expansion of $\mathcal{Q}$ does not occur in $\operatorname{Sh}(\mathcal{Q})$ is ensured by $\operatorname{Sh}(\mathcal{Q})=\operatorname{Sh}(\mathcal{P})$ and the new $\forall$-expansion having fresh eigenvariables.

\subsection{Complexity Measures}

Let $\rightarrow$ denote the reflexive, transitive closure of the mapping $\mapsto$. Our next aim is to prove weak normalization of our reduction system $\rightarrow$. It turns out that a parallel version of the proof strategy for cut-elimination can be applied to expansion trees. Equipped with this observation, we can adapt to our setting the notion of rank of a cut and the notion of maximal cut of maximal rank, which in turn will allow us to prove weak normalization. In fact, these notions can be formulated in a natural way using the language of expansion trees we have introduced so far. In sequent calculus, a maximal cut is just a cut of maximal rank having no cut of the same rank above it in the proof tree. In our setting, the geometry of the proof is represented by the dependency relation between cuts, so a maximal cut of maximal rank is just a cut of maximal rank which is not smaller, according to the dependency relation, than any cut of that rank.

Definition 11 (Rank of a Cut, Maximal Cut). Let $\mathcal{P}$ be an expansion proof with merges and $C$ a cut of $\mathcal{P}$. We define $\operatorname{rk}(C)$ as the logical complexity of $\operatorname{Sh}(C)$ and we call it the rank of $C$. We call $C$ maximal if for all cuts $D$ of $\mathcal{P}, \operatorname{rk}(D) \leqslant \operatorname{rk}(C)$ and $\operatorname{rk}(D)=\operatorname{rk}(C)$ implies that $C \nless_{\mathcal{p}} D$. 


\subsection{Weak Normalization}

This section is dedicated to proving that there exists a terminating strategy for the application of the cut-reduction rules. Given an expansion proof $\mathcal{P}$, our reduction strategy will be based on picking maximal cuts and reducing them in parallel.

Theorem 6 (Weak Normalization). For every expansion proof $\mathcal{Q}$ there is a cut-free expansion proof $\mathcal{Q}^{*}$ such that $\mathcal{Q} \rightarrow \mathcal{Q}^{*}$ and $\operatorname{Sh}(\mathcal{Q})=\operatorname{Sh}\left(\mathcal{Q}^{*}\right)$.

Proof. We partition the collection of cuts occurring in $\mathcal{Q}$ in equivalence classes, by means of the equivalence relation

$$
\begin{gathered}
C_{1} \sim C_{2} \\
\text { iff } \\
C_{1}=\left\{\exists x A+{ }^{t_{1}} E_{1} \cdots+{ }^{t_{n}} E_{n}, \forall x \bar{A}+{ }^{\alpha} F\right\} \text { and } C_{2}=\left\{\exists x A+{ }^{s_{1}} G_{1} \cdots+{ }^{s_{m}} G_{m}, \forall x \bar{A}+{ }^{\beta} H\right\}
\end{gathered}
$$

We now proceed by induction on the pair $(r, k)$, where $r$ is the greatest among the ranks of the cuts in $\mathcal{Q}$ and $k$ is the number of equivalence classes whose cuts have rank $r$. If $\mathcal{Q}$ is already cut-free, we are done. Otherwise, we wish to single out a maximal equivalence class: an equivalence class whose cuts are all maximal.

We first prove that a maximal equivalence class exists. Consider the relation $<$ between equivalence classes defined as follows: $\mathcal{A}<\mathcal{B}$ if and only if there is a $C \in \mathcal{A}$ such that $C<{ }_{\mathcal{Q}} D$ for every $D$ in $\mathcal{B}$. We begin by showing that this relation is not cyclic. Suppose indeed by contradiction that $\mathcal{A}_{1}<\ldots<\mathcal{A}_{n} \prec \mathcal{A}_{1}$. For every $i$, let $C_{i} \in \mathcal{A}_{i}$ be a cut such that $C_{i}<_{\mathcal{Q}} D$ for every $D$ in $\mathcal{A}_{i+1}$ or for every $D$ in $\mathcal{A}_{1}$, if $i=n$. By construction, $C_{1}<_{\mathcal{Q}} \ldots<_{\mathcal{Q}} C_{n}<_{\mathcal{Q}} C_{1}$, a contradiction, because $<_{\mathcal{Q}}$ is acyclic.

Secondly, we show that that for any equivalence classes $\mathcal{A}$ and $\mathcal{B}$, if we assume there are $C \in \mathcal{A}$, $D \in \mathcal{B}$ such that $C<_{\mathcal{Q}} D$, then $\mathcal{A} \prec \mathcal{B}$. Indeed, since $C<_{\mathcal{Q}} D$, we have a chain of cuts or expansions $C<_{\mathcal{Q}} w_{1}<_{\mathcal{Q}} \ldots<_{\mathcal{Q}} w_{n}<_{\mathcal{Q}} D$; moreover, by Definition $5, w_{n}$ must be a $\forall$ expansion $+{ }^{\beta}$ such that $\beta$ occurs in $\operatorname{Sh}(D)$. By definition of the relation $\sim$, for every $E \in \mathcal{B}$, we have $\operatorname{Sh}(E)=\operatorname{Sh}(D)$. Therefore, $C<_{\mathcal{Q}} w_{1}<_{\mathcal{Q}} \ldots<_{\mathcal{Q}} w_{n}<_{\mathcal{Q}} E$. We conclude $\mathcal{A}<\mathcal{B}$.

Third, suppose by contradiction that there is no maximal equivalence class, assuming there is at least one equivalence class. We want to show that for every equivalence class $\mathcal{A}$ whose cuts have rank $r$, there is an equivalence class $\mathcal{B}$ whose cuts have rank $r$ such that $\mathcal{A}<\mathcal{B}$; since there are finitely many equivalence classes, this implies that the relation $\prec$ is cyclic, a contradiction. Indeed, consider any equivalence class $\mathcal{A}$ whose cuts have rank $r$. By assumption, there is a cut $C \in \mathcal{A}$ such that $C<_{\mathcal{Q}} D$, with $D$ of rank $r$. Let $\mathcal{B}$ the equivalence class of $D$. By definition of $\sim$, all cuts of $\mathcal{B}$ have rank $r$, and by what we have proved above, $\mathcal{A}<\mathcal{B}$, which is what we wanted to show.

Now let us consider the possible reduction steps.

Quantifier Step. If there is at least a cut whose shallow formula is an existential formula, let $\sigma_{1}, \ldots, \sigma_{l}$ be respectively the substitutions $\left[t_{1} / \alpha_{1} \ldots t_{1} / \alpha_{q}\right], \ldots,\left[t_{l} / \alpha_{1} \ldots t_{l} / \alpha_{q}\right], \mathcal{A}$ be a maximal equivalence class and

$$
\begin{gathered}
\mathcal{A}=\left\{\exists x A+{ }^{t_{1}} E_{1} \cdots+{ }^{t_{n}} E_{n}, \forall x \bar{A}+{ }^{\alpha_{1}} F_{1}\right\}, \ldots,\left\{\exists x A+{ }^{t_{p}} E_{p} \cdots+{ }^{t_{l}} E_{l}, \forall x \bar{A}+{ }^{\alpha_{q}} F_{q}\right\} \\
\mathcal{Q}=\mathcal{A}, \mathcal{P} \\
\mathcal{Q}^{\prime}=\left\{E_{1}, F_{1} \eta_{1} \sigma_{1}\right\}, \ldots,\left\{E_{1}, F_{q} \eta_{1} \sigma_{1}\right\}, \ldots,\left\{E_{l}, F_{1} \eta_{l} \sigma_{l}\right\}, \ldots,\left\{E_{l}, F_{q} \eta_{l} \sigma_{l}\right\}, \mathcal{P}, \mathcal{P} \eta_{1} \sigma_{1}, \ldots, \mathcal{P} \eta_{l} \sigma_{l}
\end{gathered}
$$

where the $\forall$-expansions $+{ }^{\alpha_{1}}, \ldots,{ }^{\alpha_{q}}$ do not occur in $\mathcal{P}$, no cut in $\mathcal{P}$ has shallow formula $\exists x A$ and $\eta_{1}, \ldots, \eta_{l}$ are renamings to fresh variables of the eigenvariables $\beta$ of $\mathcal{P}, F_{1}, \ldots, F_{q}$ such that 
for some $1 \leqslant i \leqslant q$ and occurrence of $+{ }^{\alpha_{i}}$ we have $+{ }^{\alpha_{i}}<\mathcal{P}_{1}+{ }^{\beta}$. First of all, we observe that it is alway possible to satisfy the condition that no $+{ }^{\alpha_{i}}$ occurs in $\mathcal{P}$ : by weak regularity of $\mathcal{Q}$, every occurrence of $+{ }^{\alpha_{i}}$ is on the right of the shallow formula $\forall x \bar{A}$ of some cut.

Let

$$
D=\left\{\exists x B+{ }^{s_{1}} G_{1} \cdots+{ }^{s_{m}} G_{m}, \forall x \bar{B}+{ }^{\beta} H\right\}
$$

be any cut of rank $r$ in $\mathcal{P}$. We want to prove that neither $\alpha_{i}$ nor any variable $\gamma$ in the domain of any $\eta_{k}$ can occur in $\exists x B$ or be equal to $\beta$. Indeed, $\alpha_{i}$ by hypothesis must be different from $\beta$ and if it occurred in $\exists x B$, we would have by Definition 5 that for every $C \in \mathcal{A}, C<_{\mathcal{Q}}+{ }^{\alpha}<_{\mathcal{Q}} D$, contradicting the maximality of $C$. Moreover, if $\gamma$ in the domain of any $\eta_{k}$ occurred in $\exists x B$, then for some $C \in \mathcal{A}, C<_{\mathcal{Q}}+{ }^{\alpha_{i}}<_{\mathcal{Q}}+{ }^{\gamma}<_{\mathcal{Q}} D$, contradicting the maximality of $C$; on the other hand, if $\gamma$ were equal to $\beta$, then for some $C \in \mathcal{A}, C<_{\mathcal{Q}}+{ }^{\alpha_{i}}<_{\mathcal{Q}}+{ }^{\beta}$, therefore we would have a chain of expansions or cuts $+{ }^{\alpha_{i}}<_{\mathcal{Q}} w_{1}<_{\mathcal{Q}} \ldots<_{\mathcal{Q}} w_{n}<_{\mathcal{Q}}+{ }^{\beta}$, so that $w_{n}=D$, contradicting the maximality of $C$.

Let now $D_{1}, \ldots, D_{m}$ be the cuts of rank $r$ in $\mathcal{P}$. Then, for each $i$,

$$
\begin{gathered}
\mathcal{P} \eta_{i} \sigma_{i}=D_{1} \eta_{i} \sigma_{i}, \ldots, D_{m} \eta_{i} \sigma_{i}, \mathcal{P}_{i} \\
\mathcal{P}=D_{1}, \ldots, D_{m}, \mathcal{P}_{0}
\end{gathered}
$$

with no cut of rank $r$ appearing in $\mathcal{P}_{i}$. Let

$$
\mathcal{D}=D_{1}, \ldots, D_{m}, D_{1} \eta_{1} \sigma_{1}, \ldots, D_{m} \eta_{1} \sigma_{1}, \ldots, D_{1} \eta_{l} \sigma_{l}, \ldots, D_{m} \eta_{l} \sigma_{l}
$$

Then

$$
\mathcal{Q}^{\prime}=\left\{E_{1}, F_{1} \eta_{1} \sigma_{1}\right\}, \ldots,\left\{E_{1}, F_{q} \eta_{1} \sigma_{1}\right\}, \ldots,\left\{E_{l}, F_{1} \eta_{l} \sigma_{l}\right\}, \ldots,\left\{E_{l}, F_{q} \eta_{l} \sigma_{l}\right\}, \mathcal{D}, \mathcal{P}_{0}, \ldots, \mathcal{P}_{n}
$$

By what we have proved, for every $i, j$, if

$$
D_{i}=\left\{\exists x B+{ }^{s_{1}} G_{1} \cdots+{ }^{s_{m}} G_{m}, \forall x \bar{B}+{ }^{\beta} H\right\}
$$

then

$$
D_{i} \eta_{j} \sigma_{j}=\left\{\exists x B+{ }^{s_{1}^{\prime}} G_{1}^{\prime} \cdots+{ }^{s_{m}^{\prime}} G_{m}^{\prime}, \forall x \bar{B}+{ }^{\beta} H^{\prime}\right\}
$$

Therefore, the number of equivalence classes of rank $r$ in $\mathcal{Q}^{\prime}$ is the number of classes in which $\mathcal{D}$ is partitioned, that is exactly the number of classes in which $D_{1}, \ldots, D_{m}$ is partitioned: $k-1$. By induction hypothesis, $\mathcal{Q}^{\prime} \rightarrow \mathcal{Q}^{*}$, with $Q^{*}$ cut-free, which is the thesis.

Propositional Step. Assume

$$
\begin{gathered}
\mathcal{Q}=\left\{E_{1} \vee F_{1}, E_{1}^{\prime} \wedge F_{1}^{\prime}\right\}, \ldots,\left\{E_{m} \vee F_{m}, E_{m}^{\prime} \wedge F_{m}^{\prime}\right\}, \mathcal{P} \\
\mathcal{Q}^{\prime}=\left\{E_{1}, E_{1}^{\prime}\right\},\left\{F_{1}, F_{1}^{\prime}\right\}, \ldots,\left\{E_{m}, E_{m}^{\prime}\right\},\left\{F_{m}, F_{m}^{\prime}\right\}, \mathcal{P}
\end{gathered}
$$

where $\mathcal{P}$ does not contain any other cut of rank $r$. Then, the number of equivalence classes of cuts of rank $r$ in $\mathcal{Q}^{\prime}$ is strictly smaller than $k$, because propositional cuts are not in relation $\sim$ with any other cut and form singleton classes. By induction hypothesis, $\mathcal{Q}^{\prime} \rightarrow \mathcal{Q}^{*}$, with $Q^{*}$ cut-free, which is the thesis.

Atomic Step. As in the previous case. 


\subsection{Strong Normalization}

Having shown weak normalization of the cut-reduction rules in the previous section, it is important to turn to the question of strong normalization, i.e. whether all reduction sequences are of finite length. We conjecture that our cut-reduction rules are indeed strongly normalizing, and present some evidence for this claim by discussing how our reduction rules behave on a translation of the example [13, Figure 16], which shows how bridges can cause infinite loops in the setting of proof forests.

This example can be translated as an expansion proof of the form $\left(C^{+}, C^{-}\right), \top$ with $\top$ the atomic formula "true" and

$$
\begin{aligned}
& C^{+}=\exists x \overline{P(x)} \quad+{ }^{c} \overline{P(c)}+f(\alpha) \overline{P(f(\alpha))} \\
& C^{-}=\forall x P(x) \quad+{ }^{\alpha} P(\alpha)
\end{aligned}
$$

We have no issue at all:

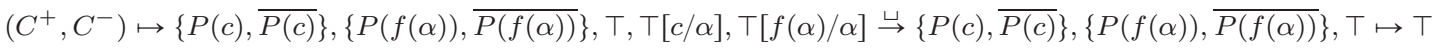

This is essentially due to the different treatment of bridges (i.e. dependencies between different sides of a cut, see Section 4.1) in our formalism: at the core of the non-termination of [13, Figure 14] lies the bridge in $\left(C^{+}, C^{-}\right)[13$, Figure 16] which induces a cycle. In the setting of proof forests, the non-termination due to bridges is handled by adding a pruning reduction, having the task of removing bridges as soon as they appear. In our setting we are able to get by naturally without pruning. This is due to our different renaming and duplication policy: not everything greater in the dependency relation than the cut is duplicated and renamed. In particular, the expansion $+^{f(\alpha)}$ is not duplicated, even if it is above $\alpha$ in the dependency relation.

\subsection{Confluence}

It is well-known that cut-elimination and similar procedures in classical logic are typically non confluent, see e.g. [24, 23, 4] for case studies and [3, 15] for asymptotic results. Neither the proof forests of [13] nor the Herbrand nets of [19] have a confluent reduction. The situation is analogous in our formalism: the reduction is not confluent. In fact, one can use the same example to demonstrate this; let

$$
\begin{aligned}
\mathcal{P}= & \left\{\exists x A+{ }^{s} A[s / x]+{ }^{t} A[t / x], \forall x \bar{A}+{ }^{\alpha} \bar{A}[\alpha / x]\right\}, \\
& \left\{\exists x B+{ }^{\alpha} B[\alpha / x], \forall x \bar{B}+{ }^{\beta} \bar{B}[\beta / x]\right\}, \\
& \exists x \exists y C+{ }^{\alpha}\left(\exists y C[\alpha / x]+{ }^{\beta} C[x / \alpha, y / \beta]\right) .
\end{aligned}
$$

which is the translation of [13, Figure 12] into an expansion proof with cut. Then it can be verified by a quick calculation that the choice of reducing either the cut on $A$ or that on $B$ first determines which of two normal forms is obtained.

However cut-elimination in classical logic can be shown confluent on the level of the (cut-free) expansion tree on a certain class of proofs [16]. For future work we hope to use such techniques for describing a confluent reduction in expansion proofs whose normal form is unique and most general in the sense that it contains all other normal forms as sub-expansions.

\section{Conclusion}

We showed in this paper that a relatively simple syntactic approach to expansion proofs with cut is possible. We strived for keeping the definitions and the technical details as elementary as 
possible. Our effort should have set the ground for addressing open combinatorial problems such as strong normalization. The price to pay for simpler reductions, however, is that we duplicate more than in Heijltjes' proof forests. This issue could be solved by an operation of merging copies of similar trees, but that should rather be understood as an optimization, rather than a theoretical necessity. Moreover, merging tends to destroy the connection with operational game semantics. In either case, however, we do not see a perfect correspondence between our cutelimination process and Coquand style plays. Copying part of the old expansion proof during the quantifier reduction step, in particular, does not seem to admit a game theoretic reading. Heijltjes' proof forests, on the contrary, avoid this copy. However, a perfect correspondence with game semantics is still a general open problem, as neither proof forests nor Herbrand nets enjoy one.

Acknowledgements. The authors would like to thank M. Baaz, K. Chaudhuri, W. Heijltjes, R. McKinley, D. Miller, and H. Moeneclaey for many helpful discussions about expansion trees, the $\varepsilon$-calculus, proof forests and Herbrand nets.

\section{References}

[1] Federico Aschieri. Game semantics and the geometry of backtracking: a new complexity analysis of interaction. Journal of Symbolic Logic, 82(2):672-708, 2017.

[2] Federico Aschieri and Margherita Zorzi. On natural deduction in classical first-order logic: Curryhoward correspondence, strong normalization and herbrand's theorem. Theor. Comput. Sci., 625:125-146, 2016.

[3] Matthias Baaz and Stefan Hetzl. On the non-confluence of cut-elimination. Journal of Symbolic Logic, 76(1):313-340, 2011.

[4] Matthias Baaz, Stefan Hetzl, Alexander Leitsch, Clemens Richter, and Hendrik Spohr. CutElimination: Experiments with CERES. In Franz Baader and Andrei Voronkov, editors, Logic for Programming, Artificial Intelligence, and Reasoning (LPAR) 2004, volume 3452 of Lecture Notes in Computer Science, pages 481-495. Springer, 2005.

[5] Matthias Baaz and Alexander Leitsch. Cut Normal Forms and Proof Complexity. Annals of Pure and Applied Logic, 97:127-177, 1999.

[6] Matthias Baaz and Alexander Leitsch. Cut-elimination and Redundancy-elimination by Resolution. Journal of Symbolic Computation, 29(2):149-176, 2000.

[7] Samuel R. Buss. On Herbrand's Theorem. In Logic and Computational Complexity, volume 960 of Lecture Notes in Computer Science, pages 195-209. Springer, 1995.

[8] Thierry Coquand. A semantics of evidence for classical arithmetic. Journal of Symbolic Logic, 60(1):325-337, 1995.

[9] Pierre-Louis Curien and Hugo Herbelin. The Duality of Computation. In Proceedings of the Fifth ACM SIGPLAN International Conference on Functional Programming (ICFP '00), pages 233-243. ACM, 2000 .

[10] Vincent Danos, Jean-Baptiste Joinet, and Harold Schellinx. A New Deconstructive Logic: Linear Logic. Journal of Symbolic Logic, 62(3):755-807, 1997.

[11] Gabriel Ebner, Stefan Hetzl, Giselle Reis, Martin Riener, Simon Wolfsteiner, and Sebastian Zivota. System description: GAPT 2.0. In Nicola Olivetti and Ashish Tiwari, editors, 8th International Joint Conference on Automated Reasoning (IJCAR), volume 9706 of Lecture Notes in Computer Science, pages 293-301. Springer, 2016.

[12] Jean-Yves Girard. Linear logic. Theoretical Computer Science, 50(1):1-101, 1987. 
[13] Willem Heijltjes. Classical proof forestry. Annals of Pure and Applied Logic, 161(11):1346-1366, 2010.

[14] Jacques Herbrand. Recherches sur la théorie de la démonstration. PhD thesis, Université de Paris, 1930.

[15] Stefan Hetzl. The Computational Content of Arithmetical Proofs. Notre Dame Journal of Formal Logic, 53(3):289-296, 2012.

[16] Stefan Hetzl and Lutz Straßburger. Herbrand-Confluence for Cut-Elimination in Classical FirstOrder Logic. In Patrick Cégielski and Arnaud Durand, editors, Computer Science Logic (CSL) 2012, volume 16 of Leibniz International Proceedings in Informatics (LIPIcs), pages 320-334. Schloss Dagstuhl-Leibniz-Zentrum fuer Informatik, 2012.

[17] David Hilbert and Paul Bernays. Grundlagen der Mathematik II. Springer, 1939.

[18] Konstantin Korovin. Instantiation-Based Automated Reasoning: From Theory to Practice. In Renate A. Schmidt, editor, 22nd International Conference on Automated Deduction (CADE), volume 5663 of Lecture Notes in Computer Science, pages 163-166. Springer, 2009.

[19] Richard McKinley. Proof nets for Herbrand's Theorem. ACM Transactions on Computational Logic, 14(1), 2013.

[20] Dale Miller. A Compact Representation of Proofs. Studia Logica, 46(4):347-370, 1987.

[21] Georg Moser and Richard Zach. The Epsilon Calculus and Herbrand Complexity. Studia Logica, 82(1):133-155, 2006.

[22] Michel Parigot. $\lambda \mu$-Calculus: An Algorithmic Interpretation of Classical Natural Deduction. In Andrei Voronkov, editor, Logic Programming and Automated Reasoning, International Conference LPAR'92, Proceedings, volume 624 of Lecture Notes in Computer Science, pages 190-201. Springer, 1992.

[23] Diana Ratiu and Trifon Trifonov. Exploring the Computational Content of the Infinite Pigeonhole Principle. 22(2):329-350, 2012. Journal of Logic and Computation.

[24] Christian Urban. Classical Logic and Computation. PhD thesis, University of Cambridge, October 2000.

[25] Christian Urban and Gavin Bierman. Strong Normalization of Cut-Elimination in Classical Logic. Fundamenta Informaticae, 45:123-155, 2000. 